\title{
Gender Distribution and Geography of Highly Cited Papers in ACS Catalysis
}

\author{
Tibor Szilvási \\ tszilvasi@ua.edu \\ Department of Chemical and Biological Engineering, The University of Alabama, Tuscaloosa, AL 35487, \\ United States
}

Following the editorial series presented in ACS Catalysis in $2020^{1-14}$, I investigated the gender distribution in highly cited ACS Catalysis papers. I adopted the structure of the Editorial series to form the studied groups of countries, and I also added two new groups: 'Small Countries' and 'Developing Countries'. As the Editorial series focused on countries from which most ACS Catalysis papers had been published, 'Small Countries' and 'Developing Countries' did not show up in the comparison. The 'Small Countries' group includes small, developed countries, such as Cyprus, Greece, Iceland, and Ireland, and due to their relatively small population, it is unrealistic for each country separately to have large contributions to ACS Catalysis. The 'Developing Countries' group captures contributions from developing countries, such as Mexico, South Africa, Thailand, and Vietnam, which also cannot individually provide a large number of papers to ACS Catalysis due to their economic status. Specific details of the methodology can be found below.

As a main result, I found that from the 155 highly cited papers that showed up in the analysis, only 15 papers have female corresponding author(s) (9.7\%), and only 5 of these 15 papers have solely female corresponding author(s) (3.2\%) (Table 1). This latter fact is in wide contrast to the large number (140) of solely male corresponding author papers. Among the 199 corresponding authors of the 155 papers analyzed here, there are only 16 female corresponding authors (8.0\%). I emphasize that out of the 16 country groups defined, I could not find female corresponding authors in 7 (Australia, Saudi Arabia, and Singapore; China; France and Spain; Germany and Netherlands; India; Japan; United States) (see details in Table 3-18). The most female corresponding authors happened to be in the groups of 'Denmark and Sweden' and 'United Kingdom' having 3-3 papers with female corresponding authors, which indicates a large concentration of papers with female corresponding authors to a few countries.

Table 1. Highly cited ACS Catalysis papers with female corresponding authors. Bold highlight indicates papers with solely female corresponding authors.

\begin{tabular}{|c|c|c|c|c|c|c|c|}
\hline Country & Author Full Names & Article Title & $\begin{array}{c}\text { Publication } \\
\text { Year }\end{array}$ & Volume & $\begin{array}{l}\text { Start } \\
\text { Page }\end{array}$ & \begin{tabular}{|c|} 
Document \\
Type
\end{tabular} & $\begin{array}{l}\text { Times } \\
\text { Cited } \\
\end{array}$ \\
\hline Czechia & $\begin{array}{l}\text { Stepankova, Veronika; Bidmanova, Sarka; Koudelakova, Tana; } \\
\text { Prokop, Zbynek; Chaloupkova, Radka; Damborsky, Jiri }\end{array}$ & Strategies for Stabilization of Enzymes in Organic Solvents & 2013 & 3 & 2823 & Article & 340 \\
\hline $\begin{array}{c}\text { Italy } \\
\text { Denmark }\end{array}$ & $\begin{array}{c}\text { Janssens, Ton V. W.; Falsig, Hanne; Lundegaard, Lars F.; } \\
\text { Vennestrom, Peter N. R.; Rasmussen, Soren B.; Moses, Poul Georg; } \\
\text { Giordanino, Filippo; Borfecchia, Elisa; Lomachenko, Kirill A.; } \\
\text { Lamberti, Carlo; Bordiga, Silvia; Godiksen, Anita; Mossin, } \\
\text { Susanne; Beato, Pablo }\end{array}$ & $\frac{\text { A Consistent Reaction Scheme for the Selective Catalytic Reduction of }}{\underline{\text { Nitrogen Oxides with Ammonia }}}$ & 2015 & 5 & 2832 & Article & 252 \\
\hline Thailand & $\begin{array}{l}\text { Huang, Yun; Handoko, Albertus D.; Hirunsit, Pussana; Yeo, Boon } \\
\text { Siang }\end{array}$ & $\begin{array}{l}\text { Electrochemical Reduction of CO2 Using Copper Single-Crystal } \\
\text { Surfaces: Effects of CO* Coverage on the Selective Formation of } \\
\text { Ethylene }\end{array}$ & 2017 & 7 & 1749 & Article & 230 \\
\hline $\begin{array}{l}\text { South } \\
\text { Korea }\end{array}$ & $\begin{array}{c}\text { Yang, Sungeun; Tak, Young Joo; Kim, Jiwhan; Soon, Aloysius; } \\
\text { Lee, Hyunjoo }\end{array}$ & $\frac{\text { Support Effects in Single-Atom Platinum Catalysts for }}{\text { Electrochemical Oxygen Reduction }}$ & 2017 & 7 & 1301 & Article & 193 \\
\hline \begin{tabular}{|c|} 
United \\
Kingdom
\end{tabular} & $\begin{array}{l}\text { France, Scott P.; Hepworth, Lorna J.; Turner, Nicholas J.; Flitsch, } \\
\text { Sabine L. }\end{array}$ & \begin{tabular}{|c|} 
Constructing Biocatalytic Cascades: In Vitro and in Vivo Approaches to \\
de Novo Multi-Enzyme Pathways
\end{tabular} & 2017 & 7 & 710 & Article & 174 \\
\hline Canada & $\begin{array}{c}\text { Klinkova, Anna; De Luna, Phil; Dinh, Cao-Thang; Voznyy, } \\
\text { Oleksandr; Larin, Egor M.; Kumacheva, Eugenia; Sargent, Edward } \\
\text { H. }\end{array}$ & \begin{tabular}{|c|}
$\frac{\text { Rational Design of Efficient Palladium Catalysts for Electroreduction of }}{\text { Carbon Dioxide to Formate }}$ \\
\end{tabular} & 2016 & 6 & 8115 & Article & 164 \\
\hline Russia & $\begin{array}{l}\text { Bulushev, Dmitri A.; Zacharska, Monika; Shlyakhova, Elena V.; } \\
\text { Chuvilin, Audrey L.; Guo, Yina; Beloshapkin, Sergey; Okotrub, } \\
\text { Alexander V.; Bulusheva, Lyubov G. }\end{array}$ & \begin{tabular}{|l}
$\frac{\text { Single Isolated Pd2+ Cations Supported on N-Doped Carbon as Active }}{\underline{\text { Sites for Hydrogen Production from Formic Acid Decomposition }}}$ \\
\end{tabular} & 2016 & 6 & 681 & Article & 141 \\
\hline Denmark & $\begin{array}{c}\text { Mirbagheri, Naghmehalsadat; Wang, Degao; Peng, Cheng; Wang, } \\
\text { Jianqiang; Huang, Qing; Fan, Chunhai; Ferapontova, Elena E. }\end{array}$ & $\begin{array}{c}\frac{\text { Visible Light Driven Photoelectrochemical Water Oxidation by } \mathrm{Zn}-\text { and }}{\text { Ti-Doped Hematite Nanostructures }} \\
\end{array}$ & 2014 & 4 & 2006 & Article & 135 \\
\hline Israel & $\begin{array}{c}\text { Davydova, Elena S.; Mukerjee, Sanjeev; Jaouen, Frederic; Dekel, } \\
\text { Dario R. }\end{array}$ & 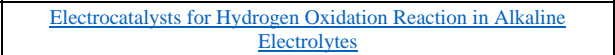 & 2018 & 8 & 6665 & Review & 126 \\
\hline
\end{tabular}




\begin{tabular}{|c|c|c|c|c|c|c|c|}
\hline $\begin{array}{c}\text { United } \\
\text { Kingdom }\end{array}$ & $\begin{array}{c}\text { Pesci, Federico M.; Sokolikova, Maria S.; Grotta, Chiara; } \\
\text { Sherrell, Peter C.; Reale, Francesco; Sharda, Kanudha; Ni, Na; } \\
\text { Palczynski, Pawel; Mattevi, Cecilia }\end{array}$ & $\frac{\text { MoS2/WS2 Heterojunction for Photoelectrochemical Water }}{\underline{\text { Oxidation }}}$ & 2017 & 7 & 4990 & Article & 112 \\
\hline Poland & $\begin{array}{c}\text { Michalow-Mauke, Katarzyna A.; Lu, Ye; Kowalski, Kazimierz; } \\
\text { Graule, Thomas; Nachtegaal, Maarten; Kroecher, Oliver; Ferri, } \\
\text { Davide }\end{array}$ & $\frac{\text { Flame-Made WO3/CeOx-TiO2 Catalysts for Selective Catalytic }}{\underline{\text { Reduction of NOx by NH3 }}}$ & 2015 & 5 & 5657 & Article & 109 \\
\hline Canada & Liu, Subiao; Liu, Qingxia; Luo, Jing-Li & \begin{tabular}{|l}
$\frac{\text { Highly Stable and Efficient Catalyst with In Situ Exsolved Fe-Ni }}{\text { Alloy Nanospheres Socketed on an Oxygen Deficient Perovskite for }}$ \\
Direct CO2 Electrolysis
\end{tabular} & 2016 & 6 & 6219 & Article & 104 \\
\hline Denmark & $\begin{array}{l}\text { Fiordaliso, Elisabetta M.; Sharafutdinov, Irek; Carvalho, } \\
\text { Hudson W. P.; Grunwaldt, Jan-D.; Hansen, Thomas W.; } \\
\text { Chorkendorff, Ib; Wagner, Jakob B.; Damsgaard, Christian D. }\end{array}$ & \begin{tabular}{|c}
$\frac{\text { Intermetallic GaPd2 Nanoparticles on SiO2 for Low-Pressure CO2 }}{\text { Hydrogenation to Methanol: Catalytic Performance and In Situ }}$ \\
Characterization
\end{tabular} & 2015 & 5 & 5827 & Article & 86 \\
\hline Mexico & $\begin{array}{c}\text { Sofia Varela, Ana; Ju, Wen; Bagger, Alexander; Franco, Patricio; } \\
\text { Rossmeisl, Jan; Strasser, Peter }\end{array}$ & $\frac{\text { Electrochemical Reduction of } \mathrm{CO} 2 \text { on Metal-Nitrogen-Doped Carbon }}{\text { Catalysts }}$ & 2019 & 9 & 7270 & Article & 83 \\
\hline
\end{tabular}

I also analyzed the total citations of the different groups (Table 2) and found that according to this methodology and metric, the 'Small Countries' and 'Developing Countries' groups had more citations than that of United Kingdom or Canada. This shows that research groups from parts of the globe that are not considered to be scientific powerhouses still provide significant contributions to ACS Catalysis.

Table 2. Total Citation of the 10 most cited papers from each groups of countries.

\section{Country Group}

United States

Australia, Saudi Arabia, and Singapore

Germany and Netherlands

China

Japan

France and Spain

Italy and Switzerland

South Korea

India

Austria, Belgium, Brazil, and Russia

Small Countries

Developing Countries

Denmark and Sweden

United Kingdom

Israel, Norway, Poland, and Taiwan

Canada

\section{Total Citation}

6973

6377

6335

5667

3373

3093

2827

2624

2412

1939

1835

1655

1488

1449

1410

1255 


\section{Methodology}

I collected the 10 most cited contributions from each group of countries using Web of Science (assessed July $9^{\text {th }}, 2021$ ) by searching for the country affiliations of the corresponding authors, resulting in a total of 160 papers (Tables 3-18). I found that 5 papers showed up twice, but I counted these papers once in the above analysis to avoid double-counting (Table 19). The gender of the corresponding authors was assessed based on their webpage/Google Scholar/ResearchGate information if otherwise not mentioned.

Table 3. Top 10 most cited ACS Catalysis papers with corresponding author affiliations from United States.

\begin{tabular}{|c|c|c|c|c|c|c|c|c|c|c|}
\hline Country & Author Full Names & Article Title & $\begin{array}{c}\text { Document } \\
\text { Type }\end{array}$ & DOI & $\begin{array}{c}\text { Publication } \\
\text { Year }\end{array}$ & Volume & $\begin{array}{l}\text { Start } \\
\text { Page }\end{array}$ & $\begin{array}{l}\text { Times } \\
\text { Cited }\end{array}$ & $\begin{array}{r}\text { Corre } \\
\text { g a } \\
(\mathrm{Men} /\end{array}$ & $\begin{array}{l}\text { ondin } \\
\text { or } \\
\text { men) }\end{array}$ \\
\hline $\begin{array}{l}\text { United } \\
\text { States }\end{array}$ & $\begin{array}{c}\text { Anantharaj, Sengeni; Ede, Sivasankara } \\
\text { Rao; Sakthikumar, Kuppan; Karthick, } \\
\text { Kannimuthu; Mishra, Soumyaranjan; } \\
\text { Kundu, Subrata } \\
\end{array}$ & \begin{tabular}{|c|} 
Recent Trends and Perspectives in \\
Electrochemical Water Splitting with an \\
Emphasis on Sulfide, Selenide, and Phosphide \\
Catalysts of $\mathrm{Fe}, \mathrm{Co}$, and Ni: A Review \\
\end{tabular} & Review & 10.1021/acscatal.6b02479 & 2016 & 6 & 8069 & 1058 & 1 & 0 \\
\hline $\begin{array}{l}\text { United } \\
\text { States }\end{array}$ & $\begin{array}{l}\text { Benck, Jesse D.; Hellstern, Thomas R.; } \\
\text { Kibsgaard, Jakob; Chakthranont, } \\
\text { Pongkarn; Jaramillo, Thomas F. }\end{array}$ & $\begin{array}{c}\text { Catalyzing the Hydrogen Evolution Reaction } \\
\text { (HER) with Molybdenum Sulfide } \\
\text { Nanomaterials }\end{array}$ & Article & $10.1021 / \mathrm{cs} 500923 \mathrm{c}$ & 2014 & 4 & 3957 & 900 & 1 & 0 \\
\hline $\begin{array}{c}\text { United } \\
\text { States }\end{array}$ & $\begin{array}{c}\text { Benck, Jesse D.; Chen, Zhebo; Kuritzky, } \\
\text { Leah Y.; Forman, Arnold J.; Jaramillo, } \\
\text { Thomas F. } \\
\end{array}$ & $\begin{array}{c}\text { Amorphous Molybdenum Sulfide Catalysts for } \\
\text { Electrochemical Hydrogen Production: Insights } \\
\text { into the Origin of their Catalytic Activity }\end{array}$ & Article & $10.1021 / \mathrm{cs} 300451 \mathrm{q}$ & 2012 & 2 & 1916 & 765 & 1 & 0 \\
\hline $\begin{array}{l}\text { United } \\
\text { States }\end{array}$ & \begin{tabular}{|c|} 
Perera, Sanjaya D.; Mariano, Ruperto G.; \\
Khiem Vu; Nour, Nijem; Seitz, Oliver; \\
Chabal, Yves; Balkus, Kenneth J., Jr. \\
\end{tabular} & $\begin{array}{c}\text { Hydrothermal Synthesis of Graphene-TiO2 } \\
\text { Nanotube Composites with Enhanced } \\
\text { Photocatalytic Activity }\end{array}$ & Article & $10.1021 / \mathrm{cs} 200621 \mathrm{c}$ & 2012 & 2 & 949 & 692 & 1 & 0 \\
\hline \begin{tabular}{l|} 
United \\
States
\end{tabular} & Grabow, L. C.; Mavrikakis, M. & $\begin{array}{l}\text { Mechanism of Methanol Synthesis on } \mathrm{Cu} \\
\text { through } \mathrm{CO} 2 \text { and } \mathrm{CO} \text { Hydrogenation }\end{array}$ & Article & $10.1021 / \mathrm{cs} 200055 \mathrm{~d}$ & 2011 & 1 & 365 & 640 & 1 & 0 \\
\hline \begin{tabular}{c|} 
United \\
States
\end{tabular} & $\begin{array}{l}\text { Kaur, Parminder; Hupp, Joseph T.; } \\
\text { Nguyen, SonBinh T. }\end{array}$ & $\begin{array}{l}\text { Porous Organic Polymers in Catalysis: } \\
\text { Opportunities and Challenges }\end{array}$ & Article & $10.1021 / \mathrm{cs} 200131 \mathrm{~g}$ & 2011 & 1 & 819 & 625 & 2 & 0 \\
\hline \begin{tabular}{l|} 
United \\
States
\end{tabular} & Liu, Jingyue & Catalysis by Supported Single Metal Atoms & Article & 10.1021/acscatal.6b01534 & 2017 & 7 & 34 & 598 & 1 & 0 \\
\hline \begin{tabular}{c|} 
United \\
States
\end{tabular} & $\begin{array}{l}\text { Wang, Jin-Liang; Wang, Cheng; Lin, } \\
\text { Wenbin }\end{array}$ & $\begin{array}{l}\text { Metal-Organic Frameworks for Light } \\
\text { Harvesting and Photocatalysis }\end{array}$ & Article & $10.1021 / \mathrm{cs} 3005874$ & 2012 & 2 & 2630 & 583 & 1 & 0 \\
\hline \begin{tabular}{c|} 
United \\
States
\end{tabular} & Nicewicz, David A.; Nguyen, Tien M. & $\begin{array}{l}\text { Recent Applications of Organic Dyes as } \\
\text { Photoredox Catalysts in Organic Synthesis }\end{array}$ & Article & $10.1021 / \mathrm{cs} 400956 \mathrm{a}$ & 2014 & 4 & 355 & 562 & 1 & 0 \\
\hline $\begin{array}{c}\text { United } \\
\text { States }\end{array}$ & \begin{tabular}{|c|} 
McKone, James R.; Sadtler, Bryce F.; \\
Werlang, Caroline A.; Lewis, Nathan S.; \\
Gray, Harry B.
\end{tabular} & $\begin{array}{l}\text { Ni-Mo Nanopowders for Efficient } \\
\text { Electrochemical Hydrogen Evolution }\end{array}$ & Article & $10.1021 / \mathrm{cs} 300691 \mathrm{~m}$ & 2013 & 3 & 166 & 550 & 1 & 0 \\
\hline
\end{tabular}

Table 4. Top 10 most cited ACS Catalysis papers with corresponding author affiliations from Australia, Saudi Arabia, and Singapore.

\begin{tabular}{|c|c|c|c|c|c|c|c|c|c|c|}
\hline Country & Author Full Names & Article Title & $\begin{array}{c}\text { Document } \\
\text { Type }\end{array}$ & DOI & $\begin{array}{c}\text { Publication } \\
\text { Year }\end{array}$ & Volume & $\begin{array}{l}\text { Start } \\
\text { Page }\end{array}$ & $\begin{array}{l}\text { Times } \\
\text { Cited }\end{array}$ & $\begin{array}{r}\text { Corr } \\
\text { a } \\
\text { Men }\end{array}$ & $\begin{array}{l}\text { inding } \\
\text { or } \\
\text { omen) }\end{array}$ \\
\hline Singapore & $\begin{array}{l}\text { Wang, Haibo; Maiyalagan, } \\
\text { Thandavarayan; Wang, Xin }\end{array}$ & $\begin{array}{c}\text { Review on Recent Progress in Nitrogen-Doped } \\
\text { Graphene: Synthesis, Characterization, and Its } \\
\text { Potential Applications }\end{array}$ & Review & $10.1021 / \operatorname{cs} 200652 y$ & 2012 & 2 & 781 & 2427 & 1 & 0 \\
\hline Singapore & $\begin{array}{c}\text { Ge, Xiaoming; Sumboja, Afriyanti; } \\
\text { Wuu, Delvin; An, Tao; Li, Bing; Goh, } \\
\text { F. W. Thomas; Hor, T. S. Andy; Zong, } \\
\text { Yun; Liu, Zhaolin }\end{array}$ & $\begin{array}{l}\text { Oxygen Reduction in Alkaline Media: From } \\
\text { Mechanisms to Recent Advances of Catalysts }\end{array}$ & Review & 10.1021/acscatal.5b00524 & 2015 & 5 & 4643 & 616 & 3 & 0 \\
\hline Singapore & $\begin{array}{c}\text { Yan, Ya; Xia, BaoYu; Xu, Zhichuan; } \\
\text { Wang, Xin }\end{array}$ & $\begin{array}{c}\begin{array}{c}\text { Recent Development of Molybdenum Sulfides } \\
\text { as Advanced Electrocatalysts for Hydrogen } \\
\text { Evolution Reaction }\end{array} \\
\end{array}$ & Review & $10.1021 / \operatorname{cs} 500070 \mathrm{x}$ & 2014 & 4 & 1693 & 594 & 1 & 0 \\
\hline Australia & $\begin{array}{c}\text { Duan, Jingjing; Chen, Sheng; Jaroniec, } \\
\text { Mietek; Qiao, Shi Zhang }\end{array}$ & $\begin{array}{l}\text { Heteroatom-Doped Graphene-Based Materials } \\
\text { for Energy-Relevant Electrocatalytic Processes }\end{array}$ & Review & 10.1021/acscatal.5b00991 & 2015 & 5 & 5207 & 565 & 1 & 0 \\
\hline Singapore & $\begin{array}{c}\text { Ren, Dan; Deng, Yilin; Handoko, } \\
\text { Albertus Denny; Chen, Chung Shou; } \\
\text { Malkhandi, Souradip; Yeo, Boon } \\
\text { Siang }\end{array}$ & $\begin{array}{c}\text { Selective Electrochemical Reduction of Carbon } \\
\text { Dioxide to Ethylene and Ethanol on Copper(I) } \\
\text { Oxide Catalysts }\end{array}$ & Article & $10.1021 / \operatorname{cs} 502128 \mathrm{q}$ & 2015 & 5 & 2814 & 450 & 1 & 0 \\
\hline Australia & $\begin{array}{l}\text { Duan, Xiaoguang; Sun, Hongqi; Wang, } \\
\text { Yuxian; Kang, Jian; Wang, Shaobin }\end{array}$ & $\begin{array}{c}\text { N-Doping-Induced Nonradical Reaction on } \\
\text { Single-Walled Carbon Nanotubes for Catalytic } \\
\text { Phenol Oxidation }\end{array}$ & Article & $10.1021 / \operatorname{cs} 5017613$ & 2015 & 5 & 553 & 400 & 2 & 0 \\
\hline Australia & $\begin{array}{c}\text { Gao, Guoping; O'Mullane, Anthony P.; } \\
\text { Du, Aijun }\end{array}$ & $\begin{array}{c}\text { 2D MXenes: A New Family of Promising } \\
\text { Catalysts for the Hydrogen Evolution Reaction }\end{array}$ & Article & 10.1021/acscatal.6b02754 & 2017 & 7 & 494 & 394 & 1 & 0 \\
\hline Australia & $\begin{array}{c}\text { Duan, Xiaoguang; Sun, Hongqi; Kang, } \\
\text { Jian; Wang, Yuxian; Indrawirawan, } \\
\text { Stacey; Wang, Shaobin }\end{array}$ & $\begin{array}{c}\text { Insights into Heterogeneous Catalysis of } \\
\text { Persulfate Activation on Dimensional-Structured } \\
\text { Nanocarbons }\end{array}$ & Article & 10.1021/acscatal.5b00774 & 2015 & 5 & 4629 & 341 & 2 & 0 \\
\hline Singapore & Takanabe, Kazuhiro & $\begin{array}{l}\text { Photocatalytic Water Splitting: Quantitative } \\
\text { Approaches toward Photocatalyst by Design }\end{array}$ & Review & 10.1021/acscatal.7b02662 & 2017 & 7 & 8006 & 280 & 1 & 0 \\
\hline $\begin{array}{c}\text { Saudi } \\
\text { Arabia }\end{array}$ & $\begin{array}{c}\text { Zhang, Jiaguang; Teo, Jason; Chen, } \\
\text { Xi; Asakura, Hiroyuki; Tanaka, } \\
\text { Tsunehiro; Teramura, Kentaro; Yan, } \\
\text { Ning }\end{array}$ & $\begin{array}{l}\text { A Series of } \mathrm{NiM}(\mathrm{M}=\mathrm{Ru}, \mathrm{Rh} \text {, and } \mathrm{Pd}) \\
\text { Bimetallic Catalysts for Effective Lignin } \\
\text { Hydrogenolysis in Water }\end{array}$ & Article & $10.1021 / \mathrm{cs} 401199 \mathrm{f}$ & 2014 & 4 & 1574 & 310 & 1 & 0 \\
\hline
\end{tabular}


Table 5. Top 10 most cited ACS Catalysis papers with corresponding author affiliations from Germany and the Netherlands.

\begin{tabular}{|c|c|c|c|c|c|c|c|c|c|c|}
\hline Country & Author Full Names & Article Title & $\begin{array}{c}\text { Document } \\
\text { Type }\end{array}$ & DOI & $\begin{array}{c}\text { Publication } \\
\text { Year }\end{array}$ & Volume & $\begin{array}{l}\text { Start } \\
\text { Page }\end{array}$ & $\begin{array}{l}\text { Times } \\
\text { Cited }\end{array}$ & $\begin{array}{r}\text { Corr } \\
\text { Men }\end{array}$ & $\begin{array}{l}\text { onding } \\
\text { or } \\
\text { omen) }\end{array}$ \\
\hline Germany & $\begin{array}{c}\text { Reier, Tobias; Oezaslan, Mehtap; } \\
\text { Strasser, Peter }\end{array}$ & \begin{tabular}{|c|} 
Electrocatalytic Oxygen Evolution Reaction \\
(OER) on Ru, Ir, and Pt Catalysts: A Comparative \\
Study of Nanoparticles and Bulk Materials \\
\end{tabular} & Article & $10.1021 / \operatorname{cs} 3003098$ & 2012 & 2 & 1765 & 1234 & 1 & 0 \\
\hline Germany & $\begin{array}{c}\text { Wang, Xinchen; Blechert, Siegfried; } \\
\text { Antonietti, Markus }\end{array}$ & $\begin{array}{c}\text { Polymeric Graphitic Carbon Nitride for } \\
\text { Heterogeneous Photocatalysis }\end{array}$ & Article & $10.1021 / \operatorname{cs} 300240 x$ & 2012 & 2 & 1596 & 1060 & 1 & 0 \\
\hline Germany & $\begin{array}{l}\text { Moselage, Marc; Li, Jie; } \\
\text { Ackermann, Lutz }\end{array}$ & Cobalt-Catalyzed C-H Activation & Review & 10.1021/acscatal.5b02344 & 2016 & 6 & 498 & 785 & 1 & 0 \\
\hline Netherlands & \begin{tabular}{|c|} 
Gascon, Jorge; Corma, Avelino; \\
Kapteijn, Freek; Llabres i Xamena, \\
Francesc X.
\end{tabular} & Metal Organic Framework Catalysis: Quo vadis? & Article & $10.1021 / \operatorname{cs} 400959 \mathrm{k}$ & 2014 & 4 & 361 & 674 & 2 & 0 \\
\hline Netherlands & $\begin{array}{c}\text { Lyaskovskyy, Volodymyr; de } \\
\text { Bruin, Bas } \\
\end{array}$ & $\begin{array}{l}\text { Redox Non-Innocent Ligands: Versatile New } \\
\text { Tools to Control Catalytic Reactions } \\
\end{array}$ & Article & $10.1021 / \operatorname{cs} 200660 \mathrm{v}$ & 2012 & 2 & 270 & 642 & 1 & 0 \\
\hline Netherlands & $\begin{array}{l}\text { Galvis, Hirsa M. Torres; de Jong, } \\
\text { Krijn P. } \\
\end{array}$ & $\begin{array}{c}\text { Catalysts for Production of Lower Olefins from } \\
\text { Synthesis Gas: A Review }\end{array}$ & Review & $10.1021 / \operatorname{cs} 4003436$ & 2013 & 3 & 2130 & 486 & 1 & 0 \\
\hline Germany & $\begin{array}{c}\text { Wunder, Stefanie; Lu, Yan; } \\
\text { Albrecht, Martin; Ballauff, Matthias }\end{array}$ & \begin{tabular}{|c|} 
Catalytic Activity of Faceted Gold Nanoparticles \\
Studied by a Model Reaction: Evidence for \\
Substrate-Induced Surface Restructuring
\end{tabular} & Article & $10.1021 / \mathrm{cs} 200208 \mathrm{a}$ & 2011 & 1 & 908 & 404 & 1 & 0 \\
\hline Germany & Liu, Weiping; Ackermann, Lutz & Manganese-Catalyzed C-H Activation & Article & 10.1021/acscatal.6b00993 & 2016 & 6 & 3743 & 374 & 1 & 0 \\
\hline Netherlands & $\begin{array}{l}\text { Diaz-Morales, Oscar; Ledezma- } \\
\text { Yanez, Isis; Koper, Marc T. M.; } \\
\text { Calle-Vallejo, Federico } \\
\end{array}$ & $\begin{array}{l}\text { Guidelines for the Rational Design of Ni-Based } \\
\text { Double Hydroxide Electrocatalysts for the } \\
\text { Oxygen Evolution Reaction }\end{array}$ & Article & 10.1021/acscatal.5b01638 & 2015 & 5 & 5380 & 338 & 2 & 0 \\
\hline Germany & Deng, Xiaohui; Tueysuez, Harun & $\begin{array}{c}\text { Cobalt-Oxide-Based Materials as Water Oxidation } \\
\text { Catalyst: Recent Progress and Challenges }\end{array}$ & Article & $10.1021 / \operatorname{cs} 500713 \mathrm{~d}$ & 2014 & 4 & 3701 & 338 & 1 & 0 \\
\hline
\end{tabular}

Table 6. Top 10 most cited ACS Catalysis papers with corresponding author affiliations from China.

\begin{tabular}{|c|c|c|c|c|c|c|c|c|c|c|}
\hline $\begin{array}{c}\text { Countr } \\
\mathbf{y}\end{array}$ & Author Full Names & Article Title & $\mid \begin{array}{c}\text { Document } \\
\text { Type }\end{array}$ & DOI & $\begin{array}{c}\text { Publication } \\
\text { Year }\end{array}$ & Volume & $\begin{array}{l}\text { Start } \\
\text { Page }\end{array}$ & $\begin{array}{l}\text { Times } \\
\text { Cited }\end{array}$ & & $\begin{array}{l}\text { inding } \\
\text { or } \\
\text { omen) }\end{array}$ \\
\hline China & $\begin{array}{l}\text { Tian, Peng; Wei, Yingxu; Ye, } \\
\text { Mao; Liu, Zhongmin }\end{array}$ & $\begin{array}{l}\text { Methanol to Olefins (MTO): From Fundamentals to } \\
\text { Commercialization }\end{array}$ & Article & $10.1021 /$ acscatal.5b00007 & 2015 & 5 & 1922 & 756 & 1 & 0 \\
\hline China & $\begin{array}{l}\text { Ye, Liqun; Liu, Jinyan; Gong, } \\
\text { Chuqing; Tian, Lihong; Peng, } \\
\text { Tianyou; Zan, Ling }\end{array}$ & $\begin{array}{c}\text { Two Different Roles of Metallic } \mathrm{Ag} \text { on } \mathrm{Ag} / \mathrm{AgX} / \mathrm{BiOX} \\
(\mathrm{X}=\mathrm{Cl}, \mathrm{Br}) \text { Visible Light Photocatalysts: Surface } \\
\text { Plasmon Resonance and Z-Scheme Bridge }\end{array}$ & Article & $10.1021 / \mathrm{cs} 300213 \mathrm{~m}$ & 2012 & 2 & 1677 & 658 & $1^{*}$ & 0 \\
\hline China & $\begin{array}{l}\text { Cheng, Daojuan; Ishihara, } \\
\text { Yoshihiro; Tan, Bin; Barbas, } \\
\text { Carlos F., III }\end{array}$ & $\begin{array}{c}\text { Organocatalytic Asymmetric Assembly Reactions: } \\
\text { Synthesis of Spirooxindoles via Organocascade } \\
\text { Strategies }\end{array}$ & Review & $10.1021 / \mathrm{cs} 401172 \mathrm{r}$ & 2014 & 4 & 743 & 624 & 1 & 0 \\
\hline China & $\begin{array}{l}\text { Shen, Kui; Chen, Xiaodong; } \\
\text { Chen, Junying; Li, Yingwei }\end{array}$ & $\begin{array}{l}\text { Development of MOF-Derived Carbon-Based } \\
\text { Nanomaterials for Efficient Catalysis }\end{array}$ & Article & 10.1021/acscatal.6b01222 & 2016 & 6 & 5887 & 623 & 1 & 0 \\
\hline China & \begin{tabular}{|c|} 
Yang, Yaqing; Zhang, Kai; Ling, \\
Huanlei; Li, Xiang; Chan, Hang \\
Cheong; Yang, Lichun; Gao, \\
Qingsheng
\end{tabular} & $\begin{array}{l}\text { MoS2-Ni3S2 Heteronanorods as Efficient and Stable } \\
\text { Bifunctional Electrocatalysts for Overall Water Splitting }\end{array}$ & Article & 10.1021/acscatal.6b03192 & 2017 & 7 & 2357 & 547 & $2^{*}$ & 0 \\
\hline China & \begin{tabular}{|c|} 
Huang, Hongwei; Li, Xiaowei; \\
Wang, Jinjian; Dong, Fan; Chu, \\
Paul K.; Zhang, Tierui; Zhang, \\
Yihe
\end{tabular} & $\begin{array}{c}\text { Anionic Group Self-Doping as a Promising Strategy: } \\
\text { Band-Gap Engineering and Multi-Functional } \\
\text { Applications of High-Performance CO32--Doped } \\
\text { Bi2O2CO3 }\end{array}$ & Article & 10.1021/acscatal.5b00444 & 2015 & 5 & 4094 & 534 & $1^{*}$ & 0 \\
\hline China & \begin{tabular}{|c|} 
Yang, Xiaofei; Cui, Haiying; Li, \\
Yang; Qin, Jieling; Zhang, \\
Rongxian; Tang, Hua \\
\end{tabular} & $\begin{array}{c}\text { Fabrication of Ag3PO4-Graphene Composites with } \\
\text { Highly Efficient and Stable Visible Light Photocatalytic } \\
\text { Performance }\end{array}$ & Article & $10.1021 / \mathrm{cs} 3008126$ & 2013 & 3 & 363 & 485 & $1^{*}$ & 0 \\
\hline China & $\begin{array}{l}\text { Li, Kan; Peng, Bosi; Peng, } \\
\text { Tianyou }\end{array}$ & $\begin{array}{c}\text { Recent Advances in Heterogeneous Photocatalytic CO2 } \\
\text { Conversion to Solar Fuels }\end{array}$ & Review & 10.1021/acscatal.6b02089 & 2016 & 6 & 7485 & 482 & 1 & 0 \\
\hline China & $\begin{array}{l}\text { Xiong, Ting; Cen, Wanglai; } \\
\text { Zhang, Yuxin; Dong, Fan }\end{array}$ & $\begin{array}{c}\text { Bridging the g-C3N4 Interlayers for Enhanced } \\
\text { Photocatalysis }\end{array}$ & Article & 10.1021/acscatal.5b02922 & 2016 & 6 & 2462 & 480 & 1 & 0 \\
\hline China & $\begin{array}{l}\text { Li, Rong; Wei, Zidong; Gou, } \\
\text { Xinglong }\end{array}$ & $\begin{array}{c}\text { Nitrogen and Phosphorus Dual-Doped Graphene/Carbon } \\
\text { Nanosheets as Bifunctional Electrocatalysts for Oxygen } \\
\text { Reduction and Evolution }\end{array}$ & Article & 10.1021/acscatal.5b00601 & 2015 & 5 & 4133 & 478 & 1 & 0 \\
\hline
\end{tabular}


Table 7. Top 10 most cited ACS Catalysis papers with corresponding author affiliations from Japan.

\begin{tabular}{|c|c|c|c|c|c|c|c|c|c|c|}
\hline Country & Author Full Names & Article Title & $\left|\begin{array}{c}\text { Document } \\
\text { Type }\end{array}\right|$ & DOI & $\begin{array}{c}\text { Publication } \\
\text { Year }\end{array}$ & Volume & $\begin{array}{l}\text { Start } \\
\text { Page }\end{array}$ & $\begin{array}{l}\text { Times } \\
\text { Cited }\end{array}$ & $\begin{array}{r}\text { Corre } \\
\text { au } \\
\text { (Men/ }\end{array}$ & \begin{tabular}{l|l} 
nding \\
men) \\
men
\end{tabular} \\
\hline Japan & Maeda, Kazuhiko & $\begin{array}{c}\text { Z-Scheme Water Splitting Using Two Different } \\
\text { Semiconductor Photocatalysts }\end{array}$ & Review & $10.1021 / \operatorname{cs} 4002089$ & 2013 & 3 & 1486 & 680 & 1 & 0 \\
\hline Japan & $\begin{array}{l}\text { Nakagawa, Yoshinao; Tamura, } \\
\text { Masazumi; Tomishige, Keiichi }\end{array}$ & $\begin{array}{c}\text { Catalytic Reduction of Biomass-Derived Furanic } \\
\text { Compounds with Hydrogen }\end{array}$ & Review & $10.1021 / \operatorname{cs} 400616 \mathrm{p}$ & 2013 & 3 & 2655 & 386 & 2 & 0 \\
\hline Japan & $\begin{array}{l}\text { Takeda, Hiroyuki; Cometto, Claudio; } \\
\text { Ishitani, Osamu; Robert, Marc }\end{array}$ & $\begin{array}{c}\text { Electrons, Photons, Protons and Earth-Abundant } \\
\text { Metal Complexes for Molecular Catalysis of } \\
\text { CO2 Reduction } \\
\end{array}$ & Review & 10.1021/acscatal.6b02181 & 2017 & 7 & 70 & 324 & 2 & 0 \\
\hline Japan & $\begin{array}{l}\text { Jiao, Jiao; Murakami, Kei; Itami, } \\
\text { Kenichiro }\end{array}$ & $\begin{array}{l}\text { Catalytic Methods for Aromatic C-H Amination: } \\
\text { An Ideal Strategy for Nitrogen-Based Functional } \\
\text { Molecules }\end{array}$ & Review & 10.1021/acscatal.5b02417 & 2016 & 6 & 610 & 323 & 1 & 0 \\
\hline Japan & \begin{tabular}{|c|} 
Liu, Yongmei; Tsunoyama, Hironori; \\
Akita, Tomoki; Xie, Songhai; Tsukuda, \\
Tatsuya \\
\end{tabular} & $\begin{array}{c}\text { Aerobic Oxidation of Cyclohexane Catalyzed by } \\
\text { Size-Controlled Au Clusters on Hydroxyapatite: } \\
\text { Size Effect in the Sub-2 nm Regime }\end{array}$ & Article & $10.1021 / \mathrm{cs} 100043 \mathrm{j}$ & 2011 & 1 & 2 & 307 & 1 & 0 \\
\hline Japan & $\begin{array}{c}\text { Shiraishi, Yasuhiro; Kanazawa, } \\
\text { Shunsuke; Sugano, Yoshitsune; } \\
\text { Tsukamoto, Daijiro; Sakamoto, } \\
\text { Hirokatsu; Ichikawa, Satoshi; Hirai, } \\
\text { Takayuki } \\
\end{array}$ & $\begin{array}{l}\text { Highly Selective Production of Hydrogen } \\
\text { Peroxide on Graphitic Carbon Nitride (g-C3N4) } \\
\text { Photocatalyst Activated by Visible Light }\end{array}$ & Article & $10.1021 / \mathrm{cs} 401208 \mathrm{c}$ & 2014 & 4 & 774 & 285 & 1 & 0 \\
\hline Japan & Obora, Yasushi & $\begin{array}{c}\text { Recent Advances in alpha-Alkylation Reactions } \\
\text { using Alcohols with Hydrogen Borrowing } \\
\text { Methodologies }\end{array}$ & Review & $10.1021 / \mathrm{cs} 501269 \mathrm{~d}$ & 2014 & 4 & 3972 & 275 & 1 & 0 \\
\hline Japan & Nakajima, Kiyotaka; Hara, Michikazu & $\begin{array}{c}\text { Amorphous Carbon with SO3H Groups as a } \\
\text { Solid Bronsted Acid Catalyst }\end{array}$ & Review & $10.1021 / \operatorname{cs} 300103 \mathrm{k}$ & 2012 & 2 & 1296 & 271 & 1 & 0 \\
\hline Japan & $\begin{array}{c}\text { Xie, Songhai; Tsunoyama, Hironori; } \\
\text { Kurashige, Wataru; Negishi, Yuichi; } \\
\text { Tsukuda, Tatsuya }\end{array}$ & $\begin{array}{c}\text { Enhancement in Aerobic Alcohol Oxidation } \\
\text { Catalysis of Au-25 Clusters by Single Pd Atom } \\
\text { Doping } \\
\end{array}$ & Article & $10.1021 / \mathrm{cs} 300252 \mathrm{~g}$ & 2012 & 2 & 1519 & 270 & 1 & 0 \\
\hline Japan & $\begin{array}{c}\text { Zhan, Wen-Wen; Zhu, Qi-Long; Xu, } \\
\text { Qjang }\end{array}$ & $\begin{array}{c}\text { Dehydrogenation of Ammonia Borane by Metal } \\
\text { Nanoparticle Catalysts }\end{array}$ & Article & 10.1021/acscatal.6b02209 & 2016 & 6 & 6892 & 252 & 1 & 0 \\
\hline
\end{tabular}

Table 8. Top 10 most cited ACS Catalysis papers with corresponding author affiliations from France and Spain.

\begin{tabular}{|c|c|c|c|c|c|c|c|c|c|c|}
\hline Country & Author Full Names & Article Title & $\mid \begin{array}{c}\text { Document } \\
\text { Type }\end{array}$ & DOI & $\begin{array}{c}\text { Publication } \\
\text { Year }\end{array}$ & Volume & $\begin{array}{l}\text { Start } \\
\text { Page }\end{array}$ & $\begin{array}{l}\text { Times } \\
\text { Cited }\end{array}$ & & $\begin{array}{l}\text { onding } \\
\text { or } \\
\text { omen) }\end{array}$ \\
\hline Spain & $\begin{array}{l}\text { Gascon, Jorge; Corma, Avelino; Kapteijn, } \\
\text { Freek; Llabres i Xamena, Francesc X. }\end{array}$ & $\begin{array}{l}\text { Metal Organic Framework Catalysis: Quo } \\
\text { vadis? }\end{array}$ & Article & $10.1021 / \operatorname{cs} 400959 \mathrm{k}$ & 2014 & 4 & 361 & 674 & 2 & 0 \\
\hline Spain & $\begin{array}{l}\text { Martin, Carmen; Fiorani, Giulia; Kleij, } \\
\text { Arian W. }\end{array}$ & \begin{tabular}{|c|} 
Recent Advances in the Catalytic Preparation \\
of Cyclic Organic Carbonates
\end{tabular} & Review & $10.1021 / \operatorname{cs} 5018997$ & 2015 & 5 & 1353 & 601 & 1 & 0 \\
\hline France & $\begin{array}{l}\text { Takeda, Hiroyuki; Cometto, Claudio; } \\
\text { Ishitani, Osamu; Robert, Marc }\end{array}$ & \begin{tabular}{|c|} 
Electrons, Photons, Protons and Earth- \\
Abundant Metal Complexes for Molecular \\
Catalysis of CO2 Reduction
\end{tabular} & Review & 10.1021/acscatal.6b02181 & 2017 & 7 & 70 & 324 & 2 & 0 \\
\hline Spain & Trovarelli, Alessandro; Llorca, Jordi & $\begin{array}{l}\text { Ceria Catalysts at Nanoscale: How Do } \\
\text { Crystal Shapes Shape Catalysis? }\end{array}$ & Article & 10.1021/acscatal.7b01246 & 2017 & 7 & 4716 & 274 & 2 & 0 \\
\hline France & $\begin{array}{l}\text { Katryniok, Benjamin; Paul, Sebastien; } \\
\text { Dumeignil, Franck }\end{array}$ & $\begin{array}{c}\text { Recent Developments in the Field of } \\
\text { Catalytic Dehydration of Glycerol to Acrolein }\end{array}$ & Review & $10.1021 / \operatorname{cs} 400354 \mathrm{p}$ & 2013 & 3 & 1819 & 216 & 1 & 0 \\
\hline Spain & $\begin{array}{c}\text { Climent, Maria J.; Corma, Avelino; Iborra, } \\
\text { Sara; Sabater, Maria J. }\end{array}$ & $\begin{array}{c}\text { Heterogeneous Catalysis for Tandem } \\
\text { Reactions }\end{array}$ & $\begin{array}{l}\text { Editorial } \\
\text { Material } \\
\end{array}$ & $10.1021 / \operatorname{cs} 401052 \mathrm{k}$ & 2014 & 4 & 870 & 212 & 1 & 0 \\
\hline France & $\begin{array}{c}\text { Zadick, Anicet; Dubau, Laetitia; Sergent, } \\
\text { Nicolas; Berthome, Gregory; Chatenet, } \\
\text { Marian }\end{array}$ & $\begin{array}{c}\text { Huge Instability of Pt/C Catalysts in Alkaline } \\
\text { Medium }\end{array}$ & Article & 10.1021/acscatal.5b01037 & 2015 & 5 & 4819 & 209 & 2 & 0 \\
\hline France & $\begin{array}{c}\text { Huan, Tran Ngoc; Ranjbar, Nastaran; } \\
\text { Rousse, Gwenaelle; Sougrati, Moulay; } \\
\text { Zitolo, Andrea; Mougel, Victor; Jaouen, } \\
\text { Frederic; Fontecave, Marc } \\
\end{array}$ & $\begin{array}{c}\text { Electrochemical Reduction of } \mathrm{CO} 2 \text { Catalyzed } \\
\text { by Fe-N-C Materials: A Structure-Selectivity } \\
\text { Study }\end{array}$ & Article & 10.1021/acscatal.6b03353 & 2017 & 7 & 1520 & 201 & 2 & 0 \\
\hline Spain & $\begin{array}{c}\text { Cordovilla, Carlos; Bartolome, Camino; } \\
\text { Martinez-Ilarduya, Jesus Ma; Espinet, Pablo }\end{array}$ & The Stille Reaction, 38 Years Later & Review & 10.1021/acscatal.5b00448 & 2015 & 5 & 3040 & 194 & 1 & 0 \\
\hline Spain & $\begin{array}{l}\text { Dhakshinamoorthy, Amarajothi; Alvaro, } \\
\text { Mercedes; Garcia, Hermenegildo }\end{array}$ & $\begin{array}{c}\text { Aerobic Oxidation of Benzylic Alcohols } \\
\text { Catalyzed by Metal-Organic Frameworks } \\
\text { Assisted by TEMPO }\end{array}$ & Article & $10.1021 / \operatorname{cs} 1000703$ & 2011 & 1 & 48 & 188 & 1 & 0 \\
\hline
\end{tabular}


Table 9. Top 10 most cited ACS Catalysis papers with corresponding author affiliations from Italy and Switzerland.

\begin{tabular}{|c|c|c|c|c|c|c|c|c|c|c|}
\hline \multirow{2}{*}{\begin{tabular}{|c|} 
Country \\
Switzerland
\end{tabular}} & \multirow{2}{*}{\begin{tabular}{|c|} 
Author Full Names \\
$\begin{array}{c}\text { Rabis, Annett; Rodriguez, } \\
\text { Paramaconi; Schmidt, Thomas J. }\end{array}$
\end{tabular}} & \multirow{2}{*}{$\begin{array}{c}\text { Article Title } \\
\begin{array}{c}\text { Electrocatalysis for Polymer Electrolyte Fuel } \\
\text { Cells: Recent Achievements and Future } \\
\text { Challenges }\end{array}\end{array}$} & \multirow{2}{*}{\begin{tabular}{|c|}
$\begin{array}{c}\text { Document } \\
\text { Type }\end{array}$ \\
Article \\
\end{tabular}} & \multirow{2}{*}{$\begin{array}{c}\text { DOI } \\
10.1021 / \mathrm{cs} 3000864\end{array}$} & \multirow{2}{*}{\begin{tabular}{|c|}
$\begin{array}{c}\text { Publication } \\
\text { Year }\end{array}$ \\
2012
\end{tabular}} & \multirow{2}{*}{\begin{tabular}{|c|} 
Volume \\
2 \\
\end{tabular}} & \multirow{2}{*}{\begin{tabular}{|r|}
$\begin{array}{l}\text { Start } \\
\text { Page }\end{array}$ \\
864
\end{tabular}} & \multirow{2}{*}{\begin{tabular}{|r|}
$\begin{array}{l}\text { Times } \\
\text { Cited }\end{array}$ \\
597 \\
\end{tabular}} & \multicolumn{2}{|c|}{$\begin{array}{c}\text { Corresponding } \\
\text { author } \\
\text { (Men/Women) }\end{array}$} \\
\hline & & & & & & & & & 1 & 0 \\
\hline Italy & Antolini, Ermete & \begin{tabular}{|c|} 
Iridium As Catalyst and Cocatalyst for Oxygen \\
Evolution/Reduction in Acidic Polymer Electrolyte \\
Membrane Electrolyzers and Fuel Cells
\end{tabular} & Review & $10.1021 / \mathrm{cs} 4011875$ & 2014 & 4 & 1426 & 327 & 1 & 0 \\
\hline Italy & \begin{tabular}{|c|} 
Aneggi, Eleonora; Wiater, Dawid; \\
de Leitenburg, Carla; Llorca, \\
Jordi; Trovarelli, Alessandro
\end{tabular} & $\begin{array}{c}\text { Shape-Dependent Activity of Ceria in Soot } \\
\text { Combustion }\end{array}$ & Article & $10.1021 / \mathrm{cs} 400850 \mathrm{r}$ & 2014 & 4 & 172 & 286 & 1 & 0 \\
\hline Italy & $\begin{array}{c}\text { Trovarelli, Alessandro; Llorca, } \\
\text { Jordi }\end{array}$ & $\begin{array}{c}\text { Ceria Catalysts at Nanoscale: How Do Crystal } \\
\text { Shapes Shape Catalysis? }\end{array}$ & Article & 10.1021/acscatal.7b01246 & 2017 & 7 & 4716 & 274 & 2 & 0 \\
\hline Italy & $\begin{array}{c}\text { Janssens, Ton V. W.; Falsig, } \\
\text { Hanne; Lundegaard, Lars F.; } \\
\text { Vennestrom, Peter N. R.; } \\
\text { Rasmussen, Soren B.; Moses, Poul } \\
\text { Georg; Giordanino, Filippo; } \\
\text { Borfecchia, Elisa; Lomachenko, } \\
\text { Kirill A.; Lamberti, Carlo; } \\
\text { Bordiga, Silvia; Godiksen, Anita; } \\
\text { Mossin, Susanne; Beato, Pablo } \\
\end{array}$ & $\begin{array}{l}\text { A Consistent Reaction Scheme for the Selective } \\
\text { Catalytic Reduction of Nitrogen Oxides with } \\
\text { Ammonia }\end{array}$ & Article & $10.1021 / \operatorname{cs} 501673 \mathrm{~g}$ & 2015 & 5 & 2832 & 252 & 1 & 2 \\
\hline Italy & Jia, Minqiang; Bandini, Marco & $\begin{array}{c}\text { Counterion Effects in Homogeneous Gold } \\
\text { Catalysis }\end{array}$ & Review & $10.1021 / \operatorname{cs} 501902 \mathrm{v}$ & 2015 & 5 & 1638 & 246 & 1 & 0 \\
\hline Italy & $\begin{array}{c}\text { Puigdollers, Antonio Ruiz; } \\
\text { Schlexer, Philomena; Tosoni, } \\
\text { Sergio; Pacchioni, Gianfranco }\end{array}$ & $\begin{array}{c}\text { Increasing Oxide Reducibility: The Role of } \\
\text { Metal/Oxide Interfaces in the Formation of } \\
\text { Oxygen Vacancies }\end{array}$ & Article & 10.1021/acscatal.7b01913 & 2017 & 7 & 6493 & 240 & 1 & 0 \\
\hline Switzerland & $\begin{array}{c}\text { Dutta, Abhijit; Rahaman, Motiar; } \\
\text { Luedi, Nicola C.; Broekmann, } \\
\text { Peter }\end{array}$ & $\begin{array}{l}\text { Morphology Matters: Tuning the Product } \\
\text { Distribution of CO2 Electroreduction on Oxide- } \\
\text { Derived Cu Foam Catalysts }\end{array}$ & Article & 10.1021/acscatal.6b00770 & 2016 & 6 & 3804 & 222 & 1 & 0 \\
\hline Switzerland & Vrubel, Heron; Hu, Xile & $\begin{array}{c}\text { Growth and Activation of an Amorphous } \\
\text { Molybdenum Sulfide Hydrogen Evolving Catalyst }\end{array}$ & Article & $10.1021 / \operatorname{cs} 400441 \mathrm{u}$ & 2013 & 3 & 2002 & 205 & 1 & 0 \\
\hline Switzerland & $\begin{array}{c}\text { Schuemperli, Martin T.; } \\
\text { Hammond, Ceri; Hermans, Ive }\end{array}$ & Developments in the Aerobic Oxidation of Amines & Review & $10.1021 / \operatorname{cs} 300212 q$ & 2012 & 2 & 1108 & 178 & 1 & 0 \\
\hline
\end{tabular}

Table 10. Top 10 most cited ACS Catalysis papers with corresponding author affiliations from South Korea.

\begin{tabular}{|c|c|c|c|c|c|c|c|c|c|c|}
\hline Country & Author Full Names & Article Title & $\begin{array}{c}\text { Document } \\
\text { Type }\end{array}$ & DOI & $\begin{array}{c}\text { Publication } \\
\text { Year }\end{array}$ & Volume & $\begin{array}{l}\text { Start } \\
\text { Page }\end{array}$ & $\begin{array}{l}\text { Times } \\
\text { Cited }\end{array}$ & $\begin{array}{r}\text { Corre } \\
\text { at } \\
(\text { Men/ }\end{array}$ & $\begin{array}{l}\text { nding } \\
\text { or } \\
\text { men) }\end{array}$ \\
\hline \begin{tabular}{l|} 
South \\
Korea
\end{tabular} & \begin{tabular}{|} 
Ganesan, Pandian; Prabu, Moni; \\
Sanetuntikul, Jakkid; \\
Shanmugam, Sangaraju
\end{tabular} & $\begin{array}{l}\text { Cobalt Sulfide Nanoparticles Grown on Nitrogen and } \\
\text { Sulfur Codoped Graphene Oxide: An Efficient } \\
\text { Electrocatalyst for Oxygen Reduction and Evolution } \\
\text { Reactions } \\
\end{array}$ & Article & 10.1021/acscatal.5b00154 & 2015 & 5 & 3625 & 458 & 1 & 0 \\
\hline $\begin{array}{l}\text { South } \\
\text { Korea }\end{array}$ & $\begin{array}{l}\text { Vij, Varun; Sultan, Siraj; } \\
\text { Harzandi, Ahmad M.; Meena, } \\
\text { Abhishek; Tiwari, Jitendra N.; } \\
\text { Lee, Wang-Geun; Yoon, } \\
\text { Taeseung; Kim, Kwang S. } \\
\end{array}$ & $\begin{array}{c}\text { Nickel-Based Electrocatalysts for Energy-Related } \\
\text { Applications: Oxygen Reduction, Oxygen Evolution, and } \\
\text { Hydrogen Evolution Reactions }\end{array}$ & Review & 10.1021/acscatal.7b01800 & 2017 & 7 & 7196 & 394 & 2 & 0 \\
\hline $\begin{array}{l}\text { South } \\
\text { Korea }\end{array}$ & \begin{tabular}{|} 
Ryu, Jaeyune; Jung, Namgee; \\
Jang, Jong Hyun; Kim, Hyoung- \\
Juhn; Yoo, Sung Jong
\end{tabular} & $\begin{array}{c}\text { In Situ Transformation of Hydrogen-Evolving CoP } \\
\text { Nanoparticles: Toward Efficient Oxygen Evolution } \\
\text { Catalysts Bearing Dispersed Morphologies with Co- } \\
\text { oxo/hydroxo Molecular Units }\end{array}$ & Article & 10.1021/acscatal.5b00349 & 2015 & 5 & 4066 & 298 & 1 & 0 \\
\hline \begin{tabular}{|l|} 
South \\
Korea \\
\end{tabular} & $\begin{array}{l}\text { Back, Seoin; Yeom, Min Sun; } \\
\text { Jung, Yousung }\end{array}$ & $\begin{array}{l}\text { Active Sites of Au and Ag Nanoparticle Catalysts for } \\
\text { CO2 Electroreduction to CO }\end{array}$ & Article & 10.1021/acscatal.5b00462 & 2015 & 5 & 5089 & 260 & 1 & 0 \\
\hline $\begin{array}{l}\text { South } \\
\text { Korea }\end{array}$ & $\begin{array}{l}\text { Yu, Xiaomei; Kim, Boseong; } \\
\text { Kim, Yu Kwon }\end{array}$ & $\begin{array}{c}\text { Highly Enhanced Photoactivity of Anatase } \mathrm{TiO} 2 \\
\text { Nanocrystals by Controlled Hydrogenation-Induced } \\
\text { Surface Defects } \\
\end{array}$ & Article & $10.1021 / \mathrm{cs} 4005776$ & 2013 & 3 & 2479 & 241 & 1 & 0 \\
\hline $\begin{array}{l}\text { South } \\
\text { Korea }\end{array}$ & $\begin{array}{c}\text { Choi, Changhyeok; Back, Seoin; } \\
\text { Kim, Na-Young; Lim, Juhyung; } \\
\text { Kim, Yong-Hyun; Jung, } \\
\text { Yousung }\end{array}$ & $\begin{array}{l}\text { Suppression of Hydrogen Evolution Reaction in } \\
\text { Electrochemical N-2 Reduction Using Single-Atom } \\
\text { Catalysts: A Computational Guideline }\end{array}$ & Article & 10.1021/acscatal.8b00905 & 2018 & 8 & 7517 & 217 & 1 & 0 \\
\hline \begin{tabular}{|l|} 
South \\
Korea \\
\end{tabular} & Mathew, Sam; Yun, Hyungdon & $\begin{array}{l}\text { omega-Transaminases for the Production of Optically } \\
\text { Pure Amines and Unnatural Amino Acids }\end{array}$ & Review & $10.1021 / \operatorname{cs} 300116 n$ & 2012 & 2 & 993 & 197 & $1 *$ & 0 \\
\hline \begin{tabular}{l|} 
South \\
Korea
\end{tabular} & Kim, Hyunwoo; Chang, Sukbok & $\begin{array}{c}\text { Transition-Metal-Mediated Direct C-H Amination of } \\
\text { Hydrocarbons with Amine Reactants: The Most } \\
\text { Desirable but Challenging C-N Bond-Formation } \\
\text { Approach }\end{array}$ & Article & 10.1021/acscatal.6b00293 & 2016 & 6 & 2341 & 195 & 1 & 0 \\
\hline \begin{tabular}{l|} 
South \\
Korea
\end{tabular} & $\begin{array}{l}\text { Yang, Sungeun; Tak, Young } \\
\text { Joo; Kim, Jiwhan; Soon, } \\
\text { Aloysius; Lee, Hyunjoo } \\
\end{array}$ & $\begin{array}{l}\text { Support Effects in Single-Atom Platinum Catalysts for } \\
\text { Electrochemical Oxygen Reduction }\end{array}$ & Article & 10.1021/acscatal.6b02899 & 2017 & 7 & 1301 & 193 & 0 & 1 \\
\hline $\begin{array}{l}\text { South } \\
\text { Korea }\end{array}$ & Patel, Pitambar; Chang, Sukbok & \begin{tabular}{|c|} 
Cobalt(III)-Catalyzed C-H Amidation of Arenes using \\
Acetoxycarbamates as Convenient Amino Sources under \\
Mild Conditions
\end{tabular} & Article & $10.1021 / \mathrm{cs} 501860 \mathrm{~b}$ & 2015 & 5 & 853 & 171 & 1 & 0 \\
\hline
\end{tabular}


Table 11. Top 10 most cited ACS Catalysis papers with corresponding author affiliations from India.

\begin{tabular}{|c|c|c|c|c|c|c|c|c|c|c|}
\hline Country & Author Full Names & Article Title & $\begin{array}{c}\text { Document } \\
\text { Type }\end{array}$ & DOI & $\begin{array}{c}\text { Publication } \\
\text { Year }\end{array}$ & Volume & $\begin{array}{l}\text { Start } \\
\text { Page }\end{array}$ & $\begin{array}{l}\text { Times } \\
\text { Cited }\end{array}$ & $\begin{array}{r}\text { Corre } \\
\text { au } \\
(\text { Men/ }\end{array}$ & $\begin{array}{l}\text { nding } \\
\text { or } \\
\text { men) }\end{array}$ \\
\hline India & $\begin{array}{c}\text { Anantharaj, Sengeni; Ede, Sivasankara } \\
\text { Rao; Sakthikumar, Kuppan; Karthick, } \\
\text { Kannimuthu; Mishra, Soumyaranjan; } \\
\text { Kundu, Subrata }\end{array}$ & \begin{tabular}{|c|} 
Recent Trends and Perspectives in \\
Electrochemical Water Splitting with an Emphasis \\
on Sulfide, Selenide, and Phosphide Catalysts of \\
Fe, Co, and Ni: A Review
\end{tabular} & Review & 10.1021/acscatal.6b02479 & 2016 & 6 & 8069 & 1058 & 1 & 0 \\
\hline India & $\begin{array}{c}\text { Chauhan, Meenakshi; Reddy, Kasala } \\
\text { Prabhakar; Gopinath, Chinnakonda S.; } \\
\text { Deka, Sasanka }\end{array}$ & $\begin{array}{l}\text { Copper Cobalt Sulfide Nanosheets Realizing a } \\
\text { Promising Electrocatalytic Oxygen Evolution } \\
\text { Reaction }\end{array}$ & Article & 10.1021/acscatal.7b01831 & 2017 & 7 & 5871 & 222 & 1 & 0 \\
\hline India & \begin{tabular}{|c|} 
Mukherjee, Sohini; Mukherjee, \\
Manjistha; Mukherjee, Arnab; Bhagi- \\
Damodaran, Ambika; Lu, Yi; Dey, \\
Abhishek
\end{tabular} & $\begin{array}{c}\text { O-2 Reduction by Biosynthetic Models of } \\
\text { Cytochrome c Oxidase: Insights into Role of } \\
\text { Proton Transfer Residues from Perturbed Active } \\
\text { Sites Models of CcO }\end{array}$ & Article & 10.1021/acscatal.8b02240 & 2018 & 8 & 8915 & 181 & 2 & 0 \\
\hline India & Deepa, Ayillath K.; Dhepe, Paresh L. & $\begin{array}{l}\text { Lignin Depolymerization into Aromatic } \\
\text { Monomers over Solid Acid Catalysts }\end{array}$ & Article & $10.1021 / \mathrm{cs} 501371 \mathrm{q}$ & 2015 & 5 & 365 & 179 & 1 & 0 \\
\hline India & $\begin{array}{l}\text { Bhowmik, Tanmay; Kundu, Manas } \\
\text { Kumar; Barman, Sudip }\end{array}$ & \begin{tabular}{|c|} 
Palladium Nanoparticle-Graphitic Carbon Nitride \\
Porous Synergistic Catalyst for Hydrogen \\
Evolution/Oxidation Reactions over a Broad \\
Range of pH and Correlation of Its Catalytic \\
Activity with Measured Hydrogen Binding \\
Energy
\end{tabular} & Article & 10.1021/acscatal.5b02485 & 2016 & 6 & 1929 & 149 & 1 & 0 \\
\hline India & $\begin{array}{c}\text { Li, Hu; Riisager, Anders; } \\
\text { Saravanamurugan, Shunmugavel; } \\
\text { Pandey, Ashok; Sangwan, Rajender } \\
\text { S.; Yang, Song; Luque, Rafael } \\
\end{array}$ & $\begin{array}{l}\text { Carbon-Increasing Catalytic Strategies for } \\
\text { Upgrading Biomass into Energy-Intensive Fuels } \\
\text { and Chemicals }\end{array}$ & Review & 10.1021/acscatal.7b02577 & 2018 & 8 & 148 & 142 & 3 & 0 \\
\hline India & Patel, Ramesh N. & $\begin{array}{c}\text { Biocatalysis: Synthesis of Key Intermediates for } \\
\text { Development of Pharmaceuticals }\end{array}$ & Review & $10.1021 / \mathrm{cs} 200219 \mathrm{~b}$ & 2011 & 1 & 1056 & 127 & 1 & 0 \\
\hline India & \begin{tabular}{|c|} 
Dhakshinamoorthy, Amarajothi; Asiri, \\
Abdullah M.; Garcia, Hermenegildo
\end{tabular} & $\begin{array}{c}\text { Metal Organic Frameworks as Versatile Hosts of } \\
\text { Au Nanoparticles in Heterogeneous Catalysis }\end{array}$ & Review & 10.1021/acscatal.6b03386 & 2017 & 7 & 2896 & 124 & 2 & 0 \\
\hline India & $\begin{array}{c}\text { Sen, Malay; Emayavaramban, } \\
\text { Balakumar; Barsu, Nagaraju; } \\
\text { Premkumar, J. Richard; Sundararaju, } \\
\text { Basker }\end{array}$ & \begin{tabular}{|c|} 
Cp*Co(III)-Catalyzed C(sp(3))-H Bond \\
Activation: A Highly Stereoselective and \\
Regioselective Alkenylation of 8-Methylquinoline \\
with Alkynes
\end{tabular} & Article & 10.1021/acscatal.6b00612 & 2016 & 6 & 2792 & 117 & 1 & 0 \\
\hline India & \begin{tabular}{|c|} 
Maity, Soham; Kancherla, Rajesh; \\
Dhawa, Uttam; Hogue, Ehtasimul; \\
Pimparkar, Sandeep; Maiti, Debabrata
\end{tabular} & \begin{tabular}{|c|}
$\begin{array}{c}\text { Switch to Allylic Selectivity in Cobalt-Catalyzed } \\
\text { Dehydrogenative Heck Reactions with Unbiased } \\
\text { Aliphatic Olefins }\end{array}$ \\
\end{tabular} & Article & 10.1021/acscatal.6b01816 & 2016 & 6 & 5493 & 113 & 1 & 0 \\
\hline
\end{tabular}

Table 12. Top 10 most cited ACS Catalysis papers with corresponding author affiliations from Austria, Belgium, Brazil, and Russia.

\begin{tabular}{|c|c|c|c|c|c|c|c|c|c|c|}
\hline Country & Author Full Names & Article Title & $\begin{array}{c}\text { Document } \\
\text { Type }\end{array}$ & DOI & $\begin{array}{c}\text { Publication } \\
\text { Year }\end{array}$ & Volume & $\begin{array}{l}\text { Start } \\
\text { Page }\end{array}$ & \begin{tabular}{|l|} 
Times \\
Cited
\end{tabular} & & $\begin{array}{l}\text { onding } \\
\text { or } \\
\text { omen) }\end{array}$ \\
\hline Russia & Ananikov, Valentine P. & $\begin{array}{l}\text { Nickel: The Spirited Horse of Transition } \\
\text { Metal Catalysis }\end{array}$ & $\begin{array}{l}\text { Editorial } \\
\text { Material }\end{array}$ & 10.1021/acscatal.5b00072 & 2015 & 5 & 1964 & 350 & 1 & 0 \\
\hline Brazil & $\begin{array}{c}\text { Scholten, Jackson D.; Leal, Barbara Caroline; } \\
\text { Dupont, Jairton }\end{array}$ & $\begin{array}{c}\text { Transition Metal Nanoparticle Catalysis in } \\
\text { Ionic Liquids }\end{array}$ & Review & $10.1021 / \operatorname{cs} 200525 \mathrm{e}$ & 2012 & 2 & 184 & 251 & 2 & 0 \\
\hline Brazil & $\begin{array}{c}\text { Lima, Carolina G. S.; Lima, Thiago de M.; } \\
\text { Duarte, Marcelo; Jurberg, Igor D.; Paixao, } \\
\text { Marcio W. }\end{array}$ & \begin{tabular}{|c|} 
Organic Synthesis Enabled by Light- \\
Irradiation of EDA Complexes: Theoretical \\
Background and Synthetic Applications \\
\end{tabular} & Review & 10.1021/acscatal.5b02386 & 2016 & 6 & 1389 & 226 & 2 & 0 \\
\hline Belgium & \begin{tabular}{|c|} 
Theofanidis, Stavros Alexandros; Galvita, \\
Vladimir V.; Poelman, Hilde; Marin, Guy B.
\end{tabular} & $\begin{array}{c}\text { Enhanced Carbon-Resistant Dry Reforming } \\
\text { Fe-Ni Catalyst: Role of Fe }\end{array}$ & Article & 10.1021/acscatal.5b00357 & 2015 & 5 & 3028 & 221 & 1 & 0 \\
\hline Austria & $\begin{array}{l}\text { Simon, Robert C.; Richter, Nina; Busto, } \\
\text { Eduardo; Kroutil, Wolfgang }\end{array}$ & \begin{tabular}{|c|} 
Recent Developments of Cascade \\
Reactions Involving omega-Transaminases
\end{tabular} & Article & $10.1021 / \mathrm{cs} 400930 \mathrm{v}$ & 2014 & 4 & 129 & 182 & 1 & 0 \\
\hline Russia & $\begin{array}{l}\text { Lyakin, Oleg Y.; Ottenbacher, Roman V.; } \\
\text { Bryliakov, Konstantin P.; Talsi, Evgenii P. }\end{array}$ & $\begin{array}{l}\text { Asymmetric Epoxidations with } \mathrm{H} 2 \mathrm{O} 2 \text { on } \\
\text { Fe and } \mathrm{Mn} \text { Aminopyridine Catalysts: } \\
\text { Probing the Nature of Active Species by } \\
\text { Combined Electron Paramagnetic } \\
\text { Resonance and Enantioselectivity Study }\end{array}$ & Article & $10.1021 / \operatorname{cs} 300205 n$ & 2012 & 2 & 1196 & 158 & 1 & 0 \\
\hline Russia & \begin{tabular}{|c|} 
Bulushev, Dmitri A.; Zacharska, Monika; \\
Lisitsyn, Alexander S.; Podyacheva, Olga \\
Yu.; Hage, Fredrik S.; Ramasse, Quentin M.; \\
Bangert, Ursel; Bulusheva, Lyubov G.
\end{tabular} & $\begin{array}{c}\text { Single Atoms of Pt-Group Metals } \\
\text { Stabilized by N-Doped Carbon Nanofibers } \\
\text { for Efficient Hydrogen Production from } \\
\text { Formic Acid }\end{array}$ & Article & 10.1021/acscatal.6b00476 & 2016 & 6 & 3442 & 161 & 2 & 0 \\
\hline Russia & \begin{tabular}{|c|} 
Bulushev, Dmitri A.; Zacharska, Monika; \\
Shlyakhova, Elena V.; Chuvilin, Audrey L.; \\
Guo, Yina; Beloshapkin, Sergey; Okotrub, \\
Alexander V.; Bulusheva, Lyubov G. \\
\end{tabular} & $\begin{array}{c}\text { Single Isolated Pd2+ Cations Supported on } \\
\text { N-Doped Carbon as Active Sites for } \\
\text { Hydrogen Production from Formic Acid } \\
\text { Decomposition }\end{array}$ & Article & 10.1021/acscatal.5b02381 & 2016 & 6 & 681 & 141 & 1 & 1 \\
\hline Belgium & \begin{tabular}{|c|} 
Dijkmans, Jan; Dusselier, Michiel; Gabriels, \\
Dries; Houthoofd, Kristof; Magusin, Pieter C. \\
M. M.; Huang, Shuigen; Pontikes, Yiannis; \\
Trekels, Maarten; Vantomme, Andre; \\
Giebeler, Lars; Oswald, Steffen; Sels, Bert F.
\end{tabular} & $\begin{array}{c}\text { Cooperative Catalysis for Multistep } \\
\text { Biomass Conversion with Sn/Al Beta } \\
\text { Zeolite }\end{array}$ & Article & $10.1021 / \mathrm{cs} 501388 \mathrm{e}$ & 2015 & 5 & 928 & 126 & 1 & 0 \\
\hline Russia & $\begin{array}{l}\text { Forslund, Robin P.; Mefford, J. Tyler; Hardin, } \\
\text { William G.; Alexander, Caleb T.; Johnston, } \\
\text { Keith P.; Stevenson, Keith J. }\end{array}$ & $\begin{array}{l}\text { Nanostructured LaNiO3 Perovskite } \\
\text { Electrocatalyst for Enhanced Urea } \\
\text { Oxidation }\end{array}$ & Article & 10.1021/acscatal.6b00487 & 2016 & 6 & 5044 & 123 & 1 & 0 \\
\hline
\end{tabular}


Table 13. Top 10 most cited ACS Catalysis papers with corresponding author affiliations from Small Countries.

\begin{tabular}{|c|c|c|c|c|c|c|c|c|c|c|}
\hline \multirow{2}{*}{\begin{tabular}{|l|} 
Country \\
Czechia
\end{tabular}} & \multirow{2}{*}{\begin{tabular}{|c|} 
Author Full Names \\
Stepankova, Veronika; Bidmanova, Sarka; \\
Koudelakova, Tana; Prokop, Zbynek; \\
Chaloupkova, Radka; Damborsky, Jiri \\
\end{tabular}} & \multirow{2}{*}{$\begin{array}{c}\text { Article Title } \\
\begin{array}{c}\text { Strategies for Stabilization of Enzymes in } \\
\text { Organic Solvents }\end{array}\end{array}$} & \multirow{2}{*}{\begin{tabular}{|c|}
$\begin{array}{c}\text { Document } \\
\text { Type }\end{array}$ \\
Article
\end{tabular}} & \multirow{2}{*}{$\begin{array}{c}\text { DOI } \\
10.1021 / \operatorname{cs} 400684 \mathrm{x}\end{array}$} & \multirow{2}{*}{\begin{tabular}{|c|}
$\begin{array}{c}\text { Publication } \\
\text { Year }\end{array}$ \\
2013
\end{tabular}} & \multirow{2}{*}{\begin{tabular}{|c|} 
Volume \\
3 \\
\end{tabular}} & \multirow{2}{*}{\begin{tabular}{|l|} 
Start \\
Page
\end{tabular}} & \multirow{2}{*}{\begin{tabular}{|c|}
$\begin{array}{c}\text { Times } \\
\text { Cited }\end{array}$ \\
340
\end{tabular}} & \multicolumn{2}{|c|}{$\begin{array}{c}\text { Corresponding } \\
\text { author } \\
\text { (Men/Women) }\end{array}$} \\
\hline & & & & & & & & & 1 & 1 \\
\hline Ireland & Lam, Edmond; Luong, John H. T. & $\begin{array}{c}\text { Carbon Materials as Catalyst Supports and } \\
\text { Catalysts in the Transformation of Biomass to } \\
\text { Fuels and Chemicals }\end{array}$ & Review & $10.1021 / \operatorname{cs} 5008393$ & 2014 & 4 & 3393 & 300 & 1 & 0 \\
\hline Iceland & $\begin{array}{c}\text { Abghoui, Younes; Garden, Anna L.; } \\
\text { Howat, Jakob G.; Vegge, Tejs; Skulason, } \\
\text { Egill }\end{array}$ & \begin{tabular}{|c|} 
Electroreduction of $\mathrm{N}-2$ to Ammonia at \\
Ambient Conditions on Mononitrides of $\mathrm{Zr}$, \\
$\mathrm{Nb}, \mathrm{Cr}$, and $\mathrm{V}: \mathrm{A}$ DFT Guide for Experiments
\end{tabular} & Article & 10.1021/acscatal.5b01918 & 2016 & 6 & 635 & 182 & 1 & 0 \\
\hline Greece & \begin{tabular}{|c|} 
Fountoulaki, Stella; Daikopoulou, \\
Vassiliki; Gkizis, Petros L.; Tamiolakis, \\
Ioannis; Armatas, Gerasimos S.; Lykakis, \\
Ioannis N. \\
\end{tabular} & $\begin{array}{l}\text { Mechanistic Studies of the Reduction of } \\
\text { Nitroarenes by NaBH4 or Hydrosilanes } \\
\text { Catalyzed by Supported Gold Nanoparticles }\end{array}$ & Article & $10.1021 / \operatorname{cs} 500379 u$ & 2014 & 4 & 3504 & 177 & 1 & 0 \\
\hline Greece & Konsolakis, Michalis & \begin{tabular}{|c|} 
Recent Advances on Nitrous Oxide (N2O) \\
Decomposition over Non-Noble-Metal Oxide \\
Catalysts: Catalytic Performance, Mechanistic \\
Considerations, and Surface Chemistry Aspects
\end{tabular} & Article & 10.1021/acscatal.5b01605 & 2015 & 5 & 6397 & 175 & 1 & 0 \\
\hline Finland & \begin{tabular}{|c|} 
Tavakkoli, Mohammad; Holmberg, Nico; \\
Kronberg, Rasmus; Jiang, Hua; Sainio, \\
Jani; Kauppinen, Esko I.; Kallio, Tanja; \\
Laasonen, Kari
\end{tabular} & $\begin{array}{l}\text { Electrochemical Activation of Single-Walled } \\
\text { Carbon Nanotubes with Pseudo-Atomic-Scale } \\
\text { Platinum for the Hydrogen Evolution Reaction }\end{array}$ & Article & 10.1021/acscatal.7b00199 & 2017 & 7 & 3121 & 153 & 2 & 0 \\
\hline Czechia & \begin{tabular}{|c|} 
Naldoni, Alberto; Altomare, Marco; \\
Zoppellaro, Giorgio; Liu, Ning; Kment, \\
Stepan; Zboril, Radek; Schmuki, Patrik
\end{tabular} & $\begin{array}{c}\text { Photocatalysis with Reduced TiO2: From } \\
\text { Black TiO2 to Cocatalyst-Free Hydrogen } \\
\text { Production }\end{array}$ & Review & 10.1021/acscatal.8b04068 & 2019 & 9 & 345 & 151 & 2 & 0 \\
\hline Slovenia & \begin{tabular}{|} 
Zabilskiy, Maxim; Djinovic, Petar; \\
Tchernychova, Elena; Tkachenko, Olga \\
P.; Kustov, Leonid M.; Pintar, Albin
\end{tabular} & $\begin{array}{l}\text { Nanoshaped } \mathrm{CuO} / \mathrm{CeO} 2 \text { Materials: Effect of } \\
\text { the Exposed Ceria Surfaces on Catalytic } \\
\text { Activity in } \mathrm{N} 2 \mathrm{O} \text { Decomposition Reaction }\end{array}$ & Article & 10.1021/acscatal.5b01044 & 2015 & 5 & 5357 & 129 & 1 & 0 \\
\hline Chechia & $\begin{array}{l}\text { Chua, Xing Juan; Luxa, Jan; Eng, Alex } \\
\text { Yong Sheng; Tan, Shu Min; Sofer, } \\
\text { Zdenek; Pumera, Martin }\end{array}$ & $\begin{array}{c}\text { Negative Electrocatalytic Effects of p-Doping } \\
\text { Niobium and Tantalum on MoS2 and WS2 for } \\
\text { the Hydrogen Evolution Reaction and Oxygen } \\
\text { Reduction Reaction }\end{array}$ & Article & 10.1021/acscatal.6b01593 & 2016 & 6 & 5724 & 114 & 1 & 0 \\
\hline Cyprus & $\begin{array}{l}\text { Kalamaras, C. M.; Dionysiou, D. D.; } \\
\text { Efstathiou, A. M. }\end{array}$ & $\begin{array}{c}\text { Mechanistic Studies of the Water-Gas Shift } \\
\text { Reaction over Pt/CexZr1-xO2 Catalysts: The } \\
\text { Effect of Pt Particle Size and Zr Dopant }\end{array}$ & Article & $10.1021 / \operatorname{cs} 3006204$ & 2012 & 2 & 2729 & 114 & 1 & 0 \\
\hline
\end{tabular}

Table 14. Top 10 most cited ACS Catalysis papers with corresponding authors from Developing Countries.

\begin{tabular}{|c|c|c|c|c|c|c|c|c|c|c|}
\hline Country & Author Full Names & Article Title & $\begin{array}{c}\text { Document } \\
\text { Type }\end{array}$ & DOI & $\begin{array}{c}\text { Publication } \\
\text { Year }\end{array}$ & Volume & $\begin{array}{l}\text { Start } \\
\text { Page }\end{array}$ & $\begin{array}{l}\text { Times } \\
\text { Cited }\end{array}$ & $\begin{array}{r}\text { Corre } \\
\text { at } \\
\text { (Men/ }\end{array}$ & $\begin{array}{l}\text { nding } \\
\text { or } \\
\text { men) }\end{array}$ \\
\hline Vietnam & $\begin{array}{l}\text { Tran, Uyen P. N.; Le, Ky K. A.; } \\
\text { Phan, Nam T. S. }\end{array}$ & \begin{tabular}{|c|} 
Expanding Applications of Metal-Organic \\
Frameworks: Zeolite Imidazolate Framework ZIF- \\
8 as an Efficient Heterogeneous Catalyst for the \\
Knoevenagel Reaction
\end{tabular} & Article & $10.1021 / \mathrm{cs} 1000625$ & 2011 & 1 & 120 & 399 & 1 & 0 \\
\hline Thailand & \begin{tabular}{|c|} 
Shaikh, Rafik Rajjak; Pornpraprom, \\
Suriyaporn; D'Elia, Valerio
\end{tabular} & \begin{tabular}{|c|} 
Catalytic Strategies for the Cycloaddition of Pure, \\
Diluted, and Waste CO2 to Epoxides under \\
Ambient Conditions
\end{tabular} & Review & 10.1021/acscatal.7b03580 & 2018 & 8 & 419 & 268 & 1 & 0 \\
\hline Thailand & $\begin{array}{l}\text { Huang, Yun; Handoko, Albertus D.; } \\
\text { Hirunsit, Pussana; Yeo, Boon Siang }\end{array}$ & $\begin{array}{c}\begin{array}{c}\text { Electrochemical Reduction of CO2 Using Copper } \\
\text { Single-Crystal Surfaces: Effects of CO* Coverage } \\
\text { on the Selective Formation of Ethylene }\end{array} \\
\end{array}$ & Article & 10.1021/acscatal.6b03147 & 2017 & 7 & 1749 & 230 & 1 & 1 \\
\hline Turkey & \begin{tabular}{|c|} 
Goksu, Haydar; Ho, Sally Fae; \\
Metin, Onder; Korkmaz, Katip; \\
Garcia, Adriana Mendoza; Gultekin, \\
Mehmet Serdar; Sun, Shouheng \\
\end{tabular} & \begin{tabular}{|c|} 
Tandem Dehydrogenation of Ammonia Borane and \\
Hydrogenation of Nitro/Nitrile Compounds \\
Catalyzed by Graphene-Supported NiPd Alloy \\
Nanoparticles
\end{tabular} & Article & $10.1021 / \mathrm{cs} 500167 \mathrm{k}$ & 2014 & 4 & 1777 & 165 & 2 & 0 \\
\hline Iran & \begin{tabular}{|c|} 
Pourjavadi, Ali; Hosseini, Seyed \\
Hassan; Doulabi, Maliheh; \\
Fakoorpoor, Seyed Mahmoud; Seidi, \\
Farzad
\end{tabular} & \begin{tabular}{|c|}
$\begin{array}{c}\text { Multi-Layer Functionalized Poly(lonic Liquid) } \\
\text { Coated Magnetic Nanoparticles: Highly } \\
\text { Recoverable and Magnetically Separable Bronsted } \\
\text { Acid Catalyst }\end{array}$ \\
\end{tabular} & Article & $10.1021 / \mathrm{cs} 300140 \mathrm{j}$ & 2012 & 2 & 1259 & 129 & 1 & 0 \\
\hline Argentina & \begin{tabular}{|c|} 
Vecchietti, Julia; Bonivardi, Adrian; \\
Xu, Wenqian; Stacchiola, Dario; \\
Delgado, Juan J.; Calatayud, \\
Monica; Collins, Sebastian E.
\end{tabular} & $\begin{array}{c}\text { Understanding the Role of Oxygen Vacancies in } \\
\text { the Water Gas Shift Reaction on Ceria-Supported } \\
\text { Platinum Catalysts }\end{array}$ & Article & $10.1021 / \mathrm{cs} 500323 \mathrm{u}$ & 2014 & 4 & 2088 & 104 & 1 & 0 \\
\hline Iran & Abedi, Sedigheh; Morsali, Ali & $\begin{array}{c}\text { Ordered Mesoporous Metal Organic Frameworks } \\
\text { Incorporated with Amorphous TiO2 As } \\
\text { Photocatalyst for Selective Aerobic Oxidation in } \\
\text { Sunlight Irradiation } \\
\end{array}$ & Article & $10.1021 / \mathrm{cs} 500123 \mathrm{~d}$ & 2014 & 4 & 1398 & 100 & 2 & 0 \\
\hline $\begin{array}{l}\text { South } \\
\text { Africa }\end{array}$ & $\begin{array}{l}\text { Xiong, Haifeng; Jewell, Linda L.; } \\
\text { Coville, Neil J. }\end{array}$ & $\begin{array}{l}\text { Shaped Carbons As Supports for the Catalytic } \\
\text { Conversion of Syngas to Clean Fuels }\end{array}$ & Review & 10.1021/acscatal.5b00090 & 2015 & 5 & 2640 & 93 & 1 & 0 \\
\hline Turkey & \begin{tabular}{|c|} 
Bulut, Ahmet; Yurderi, Mehmet; \\
Karatas, Yasar; Say, Zafer; Kivrak, \\
Hilal; Kaya, Murat; Gulcan, \\
Mehmet; Ozensoy, Emrah; \\
Zahmakiran, Mehmet \\
\end{tabular} & $\begin{array}{c}\text { MnOx-Promoted PdAg Alloy Nanoparticles for the } \\
\text { Additive-Free Dehydrogenation of Formic Acid at } \\
\text { Room Temperature }\end{array}$ & Article & 10.1021/acscatal.5b01121 & 2015 & 5 & 6099 & 84 & 1 & 0 \\
\hline Mexico & \begin{tabular}{|c|} 
Sofia Varela, Ana; Ju, Wen; Bagger, \\
Alexander; Franco, Patricio; \\
Rossmeisl, Jan; Strasser, Peter
\end{tabular} & $\begin{array}{l}\text { Electrochemical Reduction of } \mathrm{CO} 2 \text { on Metal- } \\
\text { Nitrogen-Doped Carbon Catalysts }\end{array}$ & Article & 10.1021/acscatal.9b01405 & 2019 & 9 & 7270 & 83 & 1 & 1 \\
\hline
\end{tabular}


Table 15. Top 10 most cited ACS Catalysis papers with corresponding author affiliations from Denmark and Sweden.

\begin{tabular}{|c|c|c|c|c|c|c|c|c|c|c|}
\hline Country & Author Full Names & Article Title & $\begin{array}{c}\text { Document } \\
\text { Type }\end{array} \mid$ & DOI & $\begin{array}{c}\text { Publication } \\
\text { Year }\end{array}$ & Volume & $\begin{array}{l}\text { Start } \\
\text { Page }\end{array}$ & $\begin{array}{l}\text { Times } \\
\text { Cited }\end{array}$ & $\begin{array}{r}\text { Corre } \\
\text { at } \\
\text { (Men }\end{array}$ & $\begin{array}{l}\text { nding } \\
\text { or } \\
\text { omen) }\end{array}$ \\
\hline Denmark & \begin{tabular}{|c|} 
Janssens, Ton V. W.; Falsig, Hanne; \\
Lundegaard, Lars F.; Vennestrom, Peter N. \\
R.; Rasmussen, Soren B.; Moses, Poul \\
Georg; Giordanino, Filippo; Borfecchia, \\
Elisa; Lomachenko, Kirill A.; Lamberti, \\
Carlo; Bordiga, Silvia; Godiksen, Anita; \\
Mossin, Susanne; Beato, Pablo \\
\end{tabular} & $\begin{array}{l}\text { A Consistent Reaction Scheme for the } \\
\text { Selective Catalytic Reduction of Nitrogen } \\
\text { Oxides with Ammonia }\end{array}$ & Article & $10.1021 / \mathrm{cs} 501673 \mathrm{~g}$ & 2015 & 5 & 2832 & 252 & 1 & 2 \\
\hline Denmark & $\begin{array}{l}\text { Mortensen, Peter M.; Grunwaldt, Jan-Dierk; } \\
\text { Jensen, Peter A.; Jensen, Anker D. }\end{array}$ & $\begin{array}{c}\text { Screening of Catalysts for } \\
\text { Hydrodeoxygenation of Phenol as a Model } \\
\text { Compound for Bio-oil }\end{array}$ & Article & $10.1021 / \mathrm{cs} 400266 \mathrm{e}$ & 2013 & 3 & 1774 & 252 & 1 & 0 \\
\hline Sweden & Karkas, Markus D. & \begin{tabular}{|c|} 
Photochemical Generation of Nitrogen- \\
Centered Amidyl, Hydrazonyl, and Imidyl \\
Radicals: Methodology Developments and \\
Catalytic Applications
\end{tabular} & Article & 10.1021/acscatal.7b01385 & 2017 & 7 & 4999 & 185 & 1 & 0 \\
\hline Sweden & $\begin{array}{c}\text { Yu, Fengshou; Li, Fei; Zhang, Biaobiao; Li, } \\
\text { Hua; Sun, Licheng }\end{array}$ & $\begin{array}{c}\begin{array}{c}\text { Efficient Electrocatalytic Water Oxidation } \\
\text { by a Copper Oxide Thin Film in Borate } \\
\text { Buffer }\end{array} \\
\end{array}$ & Article & $10.1021 / \mathrm{cs} 501510 \mathrm{e}$ & 2015 & 5 & 627 & 155 & 2 & 0 \\
\hline Sweden & $\begin{array}{c}\text { Hou, Jungang; Zhang, Bo; Li, Zhuwei; Cao, } \\
\text { Shuyan; Sun, Yiqing; Wu, Yunzhen; Gao, } \\
\text { Zhanming; Sun, Licheng }\end{array}$ & \begin{tabular}{|c|} 
Vertically Aligned Oxygenated-CoS2- \\
MoS2 Heteronanosheet Architecture from \\
$\begin{array}{c}\text { Polyoxometalate for Efficient and Stable } \\
\text { Overall Water Splitting }\end{array}$ \\
\end{tabular} & Article & 10.1021/acscatal.8b00668 & 2018 & 8 & 4612 & 140 & $2 *$ & 0 \\
\hline Denmark & $\begin{array}{l}\text { Mirbagheri, Naghmehalsadat; Wang, Degao; } \\
\text { Peng, Cheng; Wang, Jianqiang; Huang, } \\
\text { Qing; Fan, Chunhai; Ferapontova, Elena E. }\end{array}$ & $\begin{array}{c}\text { Visible Light Driven Photoelectrochemical } \\
\text { Water Oxidation by Zn- and Ti-Doped } \\
\text { Hematite Nanostructures } \\
\end{array}$ & Article & $10.1021 / \operatorname{cs} 500372 \mathrm{v}$ & 2014 & 4 & 2006 & 135 & 1 & 1 \\
\hline Denmark & \begin{tabular}{|c|} 
Hu, Xin-Ming; Hval, Halvor Hoen; \\
Bjerglund, Emil Tveden; Dalgaard, Kirstine \\
Junker; Madsen, Monica Rohde; Pohl, \\
Marga-Martina; Welter, Edmund; Lamagni, \\
Paolo; Buhl, Kristian Birk; Bremholm, \\
Martin; Beller, Matthias; Pedersen, Steen \\
Uttrup; Skrydstrup, Troels; Daasbjerg, Kim \\
\end{tabular} & $\begin{array}{c}\text { Selective CO2 Reduction to CO in Water } \\
\text { using Earth-Abundant Metal and Nitrogen- } \\
\text { Doped Carbon Electrocatalysts }\end{array}$ & Article & 10.1021/acscatal.8b01022 & 2018 & 8 & 6255 & 107 & 1 & 0 \\
\hline Sweden & $\begin{array}{l}\text { Ding, Xin; Gao, Yan; Zhang, Linlin; Yu, Ze; } \\
\text { Liu, Jianhui; Sun, Licheng }\end{array}$ & \begin{tabular}{|c|} 
Visible Light-Driven Water Splitting in \\
Photoelectrochemical Cells with \\
Supramolecular Catalysts on Photoanodes
\end{tabular} & Article & $10.1021 / \mathrm{cs} 500518 \mathrm{k}$ & 2014 & 4 & 2347 & 93 & 2 & 0 \\
\hline Denmark & $\begin{array}{l}\text { Fiordaliso, Elisabetta M.; Sharafutdinov, } \\
\text { Irek; Carvalho, Hudson W. P.; Grunwaldt, } \\
\text { Jan-D.; Hansen, Thomas W.; Chorkendorff, } \\
\text { Ib; Wagner, Jakob B.; Damsgaard, Christian } \\
\text { D. }\end{array}$ & $\begin{array}{c}\text { Intermetallic GaPd2 Nanoparticles on } \mathrm{SiO} 2 \\
\text { for Low-Pressure } \mathrm{CO} 2 \text { Hydrogenation to } \\
\text { Methanol: Catalytic Performance and In } \\
\text { Situ Characterization }\end{array}$ & Article & 10.1021/acscatal.5b01271 & 2015 & 5 & 5827 & 86 & 0 & 1 \\
\hline Sweden & $\begin{array}{l}\text { Behrends, Malte; Savmarker, Jonas; } \\
\text { Sjoberg, Per J. R.; Larhed, Mats }\end{array}$ & \begin{tabular}{|c|} 
Microwave-Assisted Palladium(II)- \\
Catalyzed Synthesis of Aryl Ketones from \\
Aryl Sulfinates and Direct ESI-MS Studies \\
Thereof
\end{tabular} & Article & $10.1021 / \mathrm{cs} 200428 \mathrm{u}$ & 2011 & 1 & 1455 & 83 & 1 & 0 \\
\hline
\end{tabular}

*Based on internet search

Table 16. Top 10 most cited ACS Catalysis papers with corresponding author affiliations from United Kingdom.

\begin{tabular}{|c|c|c|c|c|c|c|c|c|c|c|}
\hline Country & Author Full Names & Article Title & $\begin{array}{c}\text { Documen } \\
\text { t Type }\end{array}$ & DOI & $\begin{array}{c}\text { Publication } \\
\text { Year }\end{array}$ & Volume & $\begin{array}{l}\text { Start } \\
\text { Page }\end{array}$ & $\begin{array}{l}\text { Times } \\
\text { Cited }\end{array}$ & $\begin{array}{r}\text { Corr } \\
\text { Men } \\
\text { Ma }\end{array}$ & $\begin{array}{l}\text { onding } \\
\text { or } \\
\text { omen) }\end{array}$ \\
\hline $\begin{array}{c}\text { United } \\
\text { Kingdom }\end{array}$ & $\begin{array}{l}\text { Leitch, Jamie A.; Bhonoah, Yunas; } \\
\text { Frost, Christopher G. }\end{array}$ & $\begin{array}{c}\text { Beyond C2 and C3: Transition-Metal-Catalyzed C-H } \\
\text { Functionalization of Indole }\end{array}$ & Article & $10.1021 /$ acscatal.7b01785 & 2017 & 7 & 5618 & 205 & 1 & 0 \\
\hline $\begin{array}{c}\text { United } \\
\text { Kingdom }\end{array}$ & \begin{tabular}{|} 
Yang, Sungeun; Verdaguer- \\
Casadevall, Arnau; Arnarson, \\
Logi; Silvio, Luca; Colic, Viktor; \\
Frydendal, Rasmus; Rossmeisl, \\
Jan; Chorkendorff, Ib; Stephens, \\
Ifan E. L. \\
\end{tabular} & $\begin{array}{c}\text { Toward the Decentralized Electrochemical Production } \\
\text { of } \mathrm{H} 2 \mathrm{O} 2 \text { : A Focus on the Catalysis }\end{array}$ & Article & $10.1021 /$ acscatal.8b00217 & 2018 & 8 & 4064 & 191 & 1 & 0 \\
\hline $\begin{array}{c}\text { United } \\
\text { Kingdom }\end{array}$ & $\begin{array}{c}\text { Castro-Osma, Jose A.; Lamb, } \\
\text { Katie J.; North, Michael }\end{array}$ & \begin{tabular}{|c|} 
Cr(salophen) Complex Catalyzed Cyclic Carbonate \\
Synthesis at Ambient Temperature And Pressure
\end{tabular} & Article & 10.1021/acscatal.6b01386 & 2016 & 6 & 5012 & 167 & 2 & 0 \\
\hline $\begin{array}{c}\text { United } \\
\text { Kingdom }\end{array}$ & \begin{tabular}{|c|} 
France, Scott P.; Hepworth, Lorna \\
J.; Turner, Nicholas J.; Flitsch, \\
Sabine L.
\end{tabular} & $\begin{array}{c}\text { Constructing Biocatalytic Cascades: In Vitro and in } \\
\text { Vivo Approaches to de Novo Multi-Enzyme } \\
\text { Pathways }\end{array}$ & Article & 10.1021/acscatal.6b02979 & 2017 & 7 & 710 & 174 & 1 & 1 \\
\hline $\begin{array}{c}\text { United } \\
\text { Kingdom }\end{array}$ & $\begin{array}{l}\text { Pirez, Cyril; Caderon, Jean- } \\
\text { Michel; Dacquin, Jean-Philippe; } \\
\text { Lee, Adam F.; Wilson, Karen } \\
\end{array}$ & $\begin{array}{c}\text { Tunable KIT-6 Mesoporous Sulfonic Acid Catalysts } \\
\text { for Fatty Acid Esterification }\end{array}$ & Article & $10.1021 / \mathrm{cs} 300161 \mathrm{a}$ & 2012 & 2 & 1607 & 139 & 0 & 1 \\
\hline $\begin{array}{c}\text { United } \\
\text { Kingdom }\end{array}$ & \begin{tabular}{|c|} 
Parlett, Christopher M. A.; Bruce, \\
Duncan W.; Hondow, Nicole S.; \\
Lee, Adam F.; Wilson, Karen \\
\end{tabular} & $\begin{array}{l}\text { Support-Enhanced Selective Aerobic Alcohol } \\
\text { Oxidation over Pd/Mesoporous Silicas }\end{array}$ & Article & $10.1021 / \operatorname{cs} 200145 n$ & 2011 & 1 & 636 & 125 & 1 & 0 \\
\hline $\begin{array}{c}\text { United } \\
\text { Kingdom }\end{array}$ & \begin{tabular}{|c|} 
Yang, Bo; Burch, Robbie; \\
Hardacre, Christopher; Headdock, \\
Gareth; Hu, P.
\end{tabular} & $\begin{array}{c}\text { Origin of the Increase of Activity and Selectivity of } \\
\text { Nickel Doped by Au, Ag, and Cu for Acetylene } \\
\text { Hydrogenation }\end{array}$ & Article & $10.1021 / \mathrm{cs} 2006789$ & 2012 & 2 & 1027 & 120 & 2 & 0 \\
\hline $\begin{array}{c}\text { United } \\
\text { Kingdom }\end{array}$ & \begin{tabular}{|c|} 
Pesci, Federico M.; Sokolikova, \\
Maria S.; Grotta, Chiara; Sherrell, \\
Peter C.; Reale, Francesco; Sharda, \\
Kanudha; Ni, Na; Palczynski, \\
Pawel; Mattevi, Cecilia
\end{tabular} & $\begin{array}{c}\text { MoS2/WS2 Heterojunction for Photoelectrochemical } \\
\text { Water Oxidation }\end{array}$ & Article & $10.1021 /$ acscatal.7b01517 & 2017 & 7 & 4990 & 112 & 0 & 1 \\
\hline $\begin{array}{c}\text { United } \\
\text { Kingdom }\end{array}$ & \begin{tabular}{|c|} 
Slabu, Iustina; Galman, James L.; \\
Lloyd, Richard C.; Turner, \\
Nicholas J.
\end{tabular} & $\begin{array}{c}\text { Discovery, Engineering, and Synthetic Application of } \\
\text { Transaminase Biocatalysts }\end{array}$ & Review & 10.1021/acscatal.7b02686 & 2017 & 7 & 8263 & 110 & 1 & 0 \\
\hline $\begin{array}{c}\text { United } \\
\text { Kingdom }\end{array}$ & $\begin{array}{c}\text { Polidano, Kurt; Allen, Benjamin } \\
\text { D. W.; Williams, Jonathan M. J.; } \\
\text { Morrill, Louis C. }\end{array}$ & $\begin{array}{l}\text { Iron-Catalyzed Methylation Using the Borrowing } \\
\text { Hydrogen Approach }\end{array}$ & Article & $10.1021 /$ acscatal. 8 b02158 & 2018 & 8 & 6440 & 106 & 1 & 0 \\
\hline
\end{tabular}


Table 17. Top 10 most cited ACS Catalysis papers with corresponding author affiliations from Israel, Norway, Poland, and Taiwan.

\begin{tabular}{|c|c|c|c|c|c|c|c|c|c|c|}
\hline Country & Author Full Names & Article Title & $\begin{array}{c}\text { Document } \\
\text { Type }\end{array}$ & DOI & $\begin{array}{c}\text { Publication } \\
\text { Year }\end{array}$ & Volume & $\begin{array}{l}\text { Start } \\
\text { Page }\end{array}$ & \begin{tabular}{|l|} 
Times \\
Cited \\
\end{tabular} & $\begin{array}{r}\text { Corre } \\
\mathbf{a} \\
\text { Men }\end{array}$ & $\begin{array}{l}\text { nding } \\
\text { or } \\
\text { omen) }\end{array}$ \\
\hline Israel & Gnayem, Hani; Sasson, Yoel & \begin{tabular}{|c|} 
Hierarchical Nanostructured 3D Flowerlike \\
BiOClxBr1-x Semiconductors with Exceptional \\
Visible Light Photocatalytic Activity
\end{tabular} & Article & $10.1021 / \operatorname{cs} 3005133$ & 2013 & 3 & 186 & 215 & 1 & 0 \\
\hline Israel & Kozuch, Sebastian; Martin, Jan M. L. & Turning Over Definitions in Catalytic Cycles & $\begin{array}{l}\text { Editorial } \\
\text { Material }\end{array}$ & $10.1021 / \mathrm{cs} 3005264$ & 2012 & 2 & 2787 & 213 & 1 & 0 \\
\hline Norway & $\begin{array}{c}\text { Teketel, Shewangizaw; Skistad, } \\
\text { Wegard; Benard, Sandrine; Olsbye, } \\
\text { Unni; Lillerud, Karl Petter; Beato, } \\
\text { Pablo; Svelle, Stian }\end{array}$ & \begin{tabular}{|} 
Shape Selectivity in the Conversion of Methanol \\
to Hydrocarbons: The Catalytic Performance of \\
One-Dimensional 10-Ring Zeolites: ZSM-22, \\
ZSM-23, ZSM-48, and EU-1
\end{tabular} & Article & $10.1021 / \mathrm{cs} 200517 \mathrm{u}$ & 2012 & 2 & 26 & 151 & 1 & 0 \\
\hline Poland & \begin{tabular}{|} 
Samson, K.; Sliwa, M.; Socha, R. P.; \\
Gora-Marek, K.; Mucha, D.; \\
Rutkowska-Zbik, D.; Paul, J-F.; \\
Ruggiero-Mikolajczyk, M.; Grabowski, \\
R.; Sloczynski, J. \\
\end{tabular} & $\begin{array}{l}\text { Influence of } \mathrm{ZrO} 2 \text { Structure and Copper } \\
\text { Electronic State on Activity of } \mathrm{Cu} / \mathrm{ZrO} 2 \\
\text { Catalysts in Methanol Synthesis from CO2 }\end{array}$ & Article & $10.1021 / \mathrm{cs} 500979 \mathrm{c}$ & 2014 & 4 & 3730 & 142 & 1 & 0 \\
\hline Israel & $\begin{array}{c}\text { Davydova, Elena S.; Mukerjee, Sanjeev; } \\
\text { Jaouen, Frederic; Dekel, Dario R. }\end{array}$ & $\begin{array}{l}\text { Electrocatalysts for Hydrogen Oxidation } \\
\text { Reaction in Alkaline Electrolytes }\end{array}$ & Review & 10.1021/acscatal.8b00689 & 2018 & 8 & 6665 & 126 & 1 & 1 \\
\hline Norway & $\begin{array}{l}\text { Yang, Ming-Lei; Zhu, Yi-An; Zhou, } \\
\text { Xing-Gui; Sui, Zhi-Jun; Chen, De }\end{array}$ & $\begin{array}{l}\text { First-Principles Calculations of Propane } \\
\text { Dehydrogenation over PtSn Catalysts }\end{array}$ & Article & $10.1021 / \mathrm{cs} 300031 \mathrm{~d}$ & 2012 & 2 & 1247 & 126 & 2 & 0 \\
\hline Israel & $\begin{array}{l}\text { Hu, Peng; Diskin-Posner, Yael; Ben- } \\
\text { David, Yehoshoa; Milstein, David }\end{array}$ & \begin{tabular}{|c|} 
Reusable Homogeneous Catalytic System for \\
Hydrogen Production from Methanol and Water
\end{tabular} & Article & $10.1021 / \mathrm{cs} 500937 \mathrm{f}$ & 2014 & 4 & 2649 & 123 & 1 & 0 \\
\hline Poland & $\begin{array}{l}\text { Michalow-Mauke, Katarzyna A.; Lu, } \\
\text { Ye; Kowalski, Kazimierz; Graule, } \\
\text { Thomas; Nachtegaal, Maarten; } \\
\text { Kroecher, Oliver; Ferri, Davide }\end{array}$ & \begin{tabular}{|} 
Flame-Made WO3/CeOx-TiO2 Catalysts for \\
Selective Catalytic Reduction of NOx by NH3
\end{tabular} & Article & 10.1021/acscatal.5b01580 & 2015 & 5 & 5657 & 109 & 1 & 1 \\
\hline Israel & Gelman, Dmitri; Musa, Sanaa & $\begin{array}{c}\text { Coordination Versatility of sp(3)-Hybridized } \\
\text { Pincer Ligands toward Ligand-Metal } \\
\text { Cooperative Catalysis }\end{array}$ & Article & $10.1021 / \mathrm{cs} 3005083$ & 2012 & 2 & 2456 & 103 & 1 & 0 \\
\hline Israel & $\begin{array}{l}\text { Khusnutdinova, Julia R.; Garg, Jai } \\
\text { Anand; Milstein, David }\end{array}$ & $\begin{array}{c}\text { Combining Low-Pressure CO2 Capture and } \\
\text { Hydrogenation To Form Methanol }\end{array}$ & Article & 10.1021/acscatal.5b00194 & 2015 & 5 & 2416 & 102 & 1 & 0 \\
\hline
\end{tabular}

Table 18. Top 10 most cited ACS Catalysis papers with corresponding author affiliations from Canada.

\begin{tabular}{|c|c|c|c|c|c|c|c|c|c|c|}
\hline Country & Author Full Names & Article Title & $\begin{array}{c}\text { Document } \\
\text { Type }\end{array}$ & DOI & $\begin{array}{c}\text { Publication } \\
\text { Year }\end{array}$ & Volume & $\begin{array}{l}\text { Start } \\
\text { Page }\end{array}$ & $\begin{array}{l}\text { Times } \\
\text { Cited }\end{array}$ & $\begin{array}{r}\text { Corre } \\
\text { a } \\
\text { Men }\end{array}$ & $\begin{array}{l}\text { onding } \\
\text { or } \\
\text { omen) }\end{array}$ \\
\hline Canada & $\begin{array}{l}\text { Pitre, Spencer P.; McTiernan, Christopher } \\
\text { D.; Ismaili, Hossein; Scaiano, Juan C. }\end{array}$ & \begin{tabular}{|c|} 
Metal-Free Photocatalytic Radical \\
$\begin{array}{c}\text { Trifluoromethylation Utilizing Methylene Blue } \\
\text { and Visible Light Irradiation }\end{array}$
\end{tabular} & Article & $10.1021 / \operatorname{cs} 5005823$ & 2014 & 4 & 2530 & 165 & 1 & 0 \\
\hline Canada & $\begin{array}{c}\text { Klinkova, Anna; De Luna, Phil; Dinh, } \\
\text { Cao-Thang; Voznyy, Oleksandr; Larin, } \\
\text { Egor M.; Kumacheva, Eugenia; Sargent, } \\
\text { Edward H. }\end{array}$ & $\begin{array}{c}\text { Rational Design of Efficient Palladium } \\
\text { Catalysts for Electroreduction of Carbon } \\
\text { Dioxide to Formate }\end{array}$ & Article & 10.1021/acscatal.6b01719 & 2016 & 6 & 8115 & 164 & 1 & 1 \\
\hline Canada & $\begin{array}{c}\text { Zeng, Huiying; Qiu, Zihang; Dominguez- } \\
\text { Huerta, Alejandra; Hearne, Zoe; Chen, } \\
\text { Zhengwang; Li, Chao-Jun }\end{array}$ & $\begin{array}{c}\text { An Adventure in Sustainable Cross-Coupling } \\
\text { of Phenols and Derivatives via Carbon-Oxygen } \\
\text { Bond Cleavage }\end{array}$ & Article & 10.1021/acscatal.6b02964 & 2017 & 7 & 510 & 143 & 2 & 0 \\
\hline Canada & $\begin{array}{c}\text { Higgins, Drew C.; Hoque, Md Ariful; } \\
\text { Hassan, Fathy; Choi, Ja-Yeon; Kim, } \\
\text { Baejung; Chen, Zhongwei }\end{array}$ & $\begin{array}{c}\text { Oxygen Reduction on Graphene-Carbon } \\
\text { Nanotube Composites Doped Sequentially with } \\
\text { Nitrogen and Sulfur }\end{array}$ & Article & $10.1021 / \mathrm{cs} 5003806$ & 2014 & 4 & 2734 & 134 & 1 & 0 \\
\hline Canada & Dimitrijevic, Elena; Taylor, Mark S. & $\begin{array}{c}\text { Organoboron Acids and Their Derivatives as } \\
\text { Catalysts for Organic Synthesis }\end{array}$ & Review & $10.1021 / \operatorname{cs} 4000848$ & 2013 & 3 & 945 & 113 & 1 & 0 \\
\hline Canada & $\begin{array}{c}\text { Li, Wenmu; Wu, Jason; Higgins, Drew C.; } \\
\text { Choi, Ja-Yeon; Chen, Zhongwei }\end{array}$ & $\begin{array}{c}\text { Determination of Iron Active Sites in } \\
\text { Pyrolyzed Iron-Based Catalysts for the Oxygen } \\
\text { Reduction Reaction }\end{array}$ & Article & $10.1021 / \operatorname{cs} 300579 b$ & 2012 & 2 & 2761 & 113 & 1 & 0 \\
\hline Canada & $\begin{array}{c}\text { Nair, Mahesh Muraleedharan; Kaliaguine, } \\
\text { Serge; Kleitz, Freddy }\end{array}$ & $\begin{array}{c}\text { Nanocast LaNiO3 Perovskites as Precursors for } \\
\text { the Preparation of Coke-Resistant Dry } \\
\text { Reforming Catalysts }\end{array}$ & Article & $10.1021 / \mathrm{cs} 500918 \mathrm{c}$ & 2014 & 4 & 3837 & 110 & 2 & 0 \\
\hline Canada & \begin{tabular}{|c|} 
Balcha, Tesfalidet; Strobl, Jonathan R.; \\
Fowler, Candace; Dash, Priyabrat; Scott, \\
Robert W. J.
\end{tabular} & $\begin{array}{c}\text { Selective Aerobic Oxidation of Crotyl Alcohol } \\
\text { Using AuPd Core-Shell Nanoparticles }\end{array}$ & Article & $10.1021 / \mathrm{cs} 200040 \mathrm{a}$ & 2011 & 1 & 425 & 106 & 1 & 0 \\
\hline Canada & Liu, Subiao; Liu, Qingxia; Luo, Jing-Li & $\begin{array}{l}\text { Highly Stable and Efficient Catalyst with In } \\
\text { Situ Exsolved Fe-Ni Alloy Nanospheres } \\
\text { Socketed on an Oxygen Deficient Perovskite } \\
\text { for Direct CO2 Electrolysis }\end{array}$ & Article & 10.1021/acscatal.6b01555 & 2016 & 6 & 6219 & 104 & 0 & 1 \\
\hline Canada & $\begin{array}{l}\text { Yen, Hoang; Seo, Yongbeom; Kaliaguine, } \\
\text { Serge; Kleitz, Freddy }\end{array}$ & $\begin{array}{l}\text { Role of Metal-Support Interactions, Particle } \\
\text { Size, and Metal-Metal Synergy in CuNi } \\
\text { Nanocatalysts for H-2 Generation }\end{array}$ & Article & 10.1021/acscatal.5b00869 & 2015 & 5 & 5505 & 103 & 1 & 0 \\
\hline
\end{tabular}


Table 19. Top cited ACS Catalysis papers shown twice in Table 3-18 due to corresponding author affiliations from two different country groups.

\begin{tabular}{|c|c|c|c|c|c|c|c|c|c|c|}
\hline Country & Author Full Names & Article Title & $\begin{array}{c}\text { Document } \\
\text { Type }\end{array}$ & DOI & $\begin{array}{c}\text { Publication } \\
\text { Year }\end{array}$ & Volume & $\begin{array}{l}\text { Start } \\
\text { Page }\end{array}$ & $\begin{array}{l}\text { Times } \\
\text { Cited }\end{array}$ & & $\begin{array}{l}\text { nding } \\
\text { or } \\
\text { men) }\end{array}$ \\
\hline $\begin{array}{l}\text { India } \\
\text { United } \\
\text { States } \\
\end{array}$ & $\begin{array}{c}\text { Anantharaj, Sengeni; Ede, Sivasankara } \\
\text { Rao; Sakthikumar, Kuppan; Karthick, } \\
\text { Kannimuthu; Mishra, Soumyaranjan; } \\
\text { Kundu, Subrata }\end{array}$ & \begin{tabular}{|c|} 
Recent Trends and Perspectives in \\
Electrochemical Water Splitting with an \\
Emphasis on Sulfide, Selenide, and Phosphide \\
Catalysts of $\mathrm{Fe}, \mathrm{Co}$, and Ni: A Review \\
\end{tabular} & Review & 10.1021/acscatal.6b02479 & 2016 & 6 & 8069 & 1058 & 1 & 0 \\
\hline $\begin{array}{c}\text { Netherlands } \\
\text { Spain }\end{array}$ & $\begin{array}{c}\text { Gascon, Jorge; Corma, Avelino; } \\
\text { Kapteijn, Freek; Llabres i Xamena, } \\
\text { Francesc X. }\end{array}$ & $\begin{array}{l}\text { Metal Organic Framework Catalysis: Quo } \\
\text { vadis? }\end{array}$ & Article & $10.1021 / \mathrm{cs} 400959 \mathrm{k}$ & 2014 & 4 & 361 & 674 & 2 & 0 \\
\hline $\begin{array}{l}\text { France } \\
\text { Japan }\end{array}$ & $\begin{array}{l}\text { Takeda, Hiroyuki; Cometto, Claudio; } \\
\text { Ishitani, Osamu; Robert, Marc }\end{array}$ & $\begin{array}{l}\text { Electrons, Photons, Protons and Earth- } \\
\text { Abundant Metal Complexes for Molecular } \\
\text { Catalysis of CO2 Reduction }\end{array}$ & Review & 10.1021/acscatal.6b02181 & 2017 & 7 & 70 & 324 & 2 & 0 \\
\hline $\begin{array}{l}\text { Italy } \\
\text { Spain } \\
\end{array}$ & Trovarelli, Alessandro; Llorca, Jordi & $\begin{array}{c}\text { Ceria Catalysts at Nanoscale: How Do Crystal } \\
\text { Shapes Shape Catalysis? }\end{array}$ & Article & 10.1021/acscatal.7b01246 & 2017 & 7 & 4716 & 274 & 2 & 0 \\
\hline $\begin{array}{l}\text { Denmark } \\
\text { Italy }\end{array}$ & \begin{tabular}{|c|} 
Janssens, Ton V. W.; Falsig, Hanne; \\
Lundegaard, Lars F.; Vennestrom, Peter \\
N. R.; Rasmussen, Soren B.; Moses, \\
Poul Georg; Giordanino, Filippo; \\
Borfecchia, Elisa; Lomachenko, Kirill \\
A.; Lamberti, Carlo; Bordiga, Silvia; \\
Godiksen, Anita; Mossin, Susanne; \\
Beato, Pablo \\
\end{tabular} & $\begin{array}{l}\text { A Consistent Reaction Scheme for the } \\
\text { Selective Catalytic Reduction of Nitrogen } \\
\text { Oxides with Ammonia }\end{array}$ & Article & $10.1021 / \operatorname{cs} 501673 \mathrm{~g}$ & 2015 & 5 & 2832 & 252 & 1 & 2 \\
\hline
\end{tabular}

\section{References}

1. Jones, C.W. ACS Catalysis Highlights Its Most Cited Papers from Around the Globe: China. ACS Catalysis 10, 2762-2763 (2020).

2. Jones, C.W. ACS Catalysis Highlights Its Most Cited Papers from Around the Globe: Italy and Switzerland. ACS Catalysis 10, 3514-3515 (2020).

3. Jones, C.W. ACS Catalysis Highlights Its Most Cited Papers From Around the Globe: Canada. ACS Catalysis 10, 3807-3808 (2020).

4. Jones, C.W. ACS Catalysis Highlights Its Most Cited Papers from Around the Globe: Republic of Korea. ACS Catalysis 10, 5371-5371 (2020).

5. Jones, C.W. ACS Catalysis Highlights Its Most Cited Papers from Around the Globe: France and Spain. ACS Catalysis 10, 6473-6474 (2020).

6. Jones, C.W. ACS Catalysis Highlights Its Most Cited Papers from Around the Globe: India. ACS Catalysis 10, 6786-6787 (2020).

7. Jones, C.W. ACS Catalysis Highlights Its Most Cited Papers from Around the Globe: Austria, Belgium, Brazil, and Russia. ACS Catalysis 10, $7932-7933$ (2020).

8. Jones, C.W. ACS Catalysis Reflects on the 2019 Journal Impact Factor and Highlights Its Most Cited Papers from Around the Globe: Israel, Norway, Poland, and Taiwan. ACS Catalysis 10, 86488649 (2020).

9. Gunnoe, T.B. ACS Catalysis Highlights Its Most Cited Papers from Around the Globe: Australia, Saudi Arabia, and Singapore. ACS Catalysis 10, 10125-10126 (2020).

10. Gunnoe, T.B. ACS Catalysis Highlights Its Most Cited Papers from Around the Globe: Japan. ACS Catalysis 10, 10715-10716 (2020).

11. Gunnoe, T.B. ACS Catalysis Highlights Its Most Cited Papers from Around the Globe: United Kingdom. ACS Catalysis 10, 11663-11664 (2020).

12. Gunnoe, T.B. ACS Catalysis Highlights Its Most Cited Papers from Around the Globe: Denmark and Sweden. ACS Catalysis 10, 12340-12341 (2020).

13. Gunnoe, T.B. ACS Catalysis Highlights Its Most Cited Papers from Around the Globe: Germany and The Netherlands. ACS Catalysis 10, 13549-13550 (2020).

14. Gunnoe, T.B. ACS Catalysis Highlights Its Most Cited Papers From Around the Globe: United States. ACS Catalysis 10, 15140-15141 (2020). 